\title{
BALLOONS, DOMES AND GEOMETRY
}

\author{
IVAÏLO M. MLADENOV AND JOHN OPREA
}

Presented by Ivaïlo M. Mladenov

Abstract. In this survey, we present physical and mathematical ideas surrounding the geometry of balloons and of pneumatic domes.

\section{Introduction}

The fact that geometric principles and tools are fundamental to structural engineering is well-appreciated in the engineering world, but little-known in Mathematics. The point of the present work is to survey the types of mathematical and physical arguments that arise in the study of inflatable membranes in an attempt to make the subject accessible to mathematicians. In previous works (see [19, 20]), we have considered the shape of a Mylar balloon in the context of surface theory. While there we presented some physical arguments that we hoped would place the balloon in a broader context, here we want to carry this further to show the beautiful connections between structure and geometry. Of course, we are not the first to do this. The bibliography contains references (such as $[2-5,9,13,15,17,23,24]$ ) that will at least start the reader on the path to investigating an area where differential geometry plays a leading role in our understanding of practical engineering problems.

Membranes are a special class of structures; they are made up of thin "films" which carry load (almost) exclusively in tension (due to a lack of bending stiffness). Examples of membranes include soap bubbles and balloons (especially Mylar balloons and large scientific balloons). With the advent of new technologies, membranes have found a place in structural engineering as designs for pneumatic domes (enclosing arenas for instance). Inflated membranes are structures where the membrane remains in place due to some internal pressure. Thus, the forces acting on an inflated membrane are the pressure difference across the membrane and the tension in the membrane (as in a soap film or bubble).

For many years, NASA has been interested in inflatable membranes because they have small mass and can be transported while deflated — thus occupying a small 
volume. Indeed, there are projects investigating whether inflatable membranes can act as antennas about Mars. Materials for inflatable membranes are usually thin polymers (which have very small bending stiffness). As mentioned previously, this means that membranes carry load in tension alone. The structural behavior of membranes depends on their geometries and that is why understanding the connection between geometry and physical processes is crucial in inflatable membrane design.

\section{Tensions and Geometry}

\subsection{Membrane Geometry}

Our membrane will be modeled by a surface of revolution about the $z$-axis generated by a profile curve $(r(s), z(s))$ in the (first quadrant of the) $x z$-plane (where $s$ is the arclength parameter and we take $z(s)$ to increase with increasing $s: z(s)$ rising from the $x$-axis and meeting the $z$-axis orthogonally). This surface has a parameterization

$$
\mathbf{x}(s, v)=(r(s) \cos v, r(s) \sin v, z(s))=r(s) \mathbf{e}_{1}(v)+z(s) \mathbf{e}_{3}(v)
$$

where the unit radial vector is $\mathbf{e}_{1}(v)=\cos v \mathbf{i}+\sin v \mathbf{j}$ and $\mathbf{e}_{3}(v)=\mathbf{k}$. We also take $\mathbf{e}_{2}(v)=\mathbf{k} \times \mathbf{e}_{1}=-\sin v \mathbf{i}+\cos v \mathbf{j}$, the unit vector along the parallels of revolution. A meridian $r(s) \mathbf{e}_{1}(\stackrel{i}{v})+z(s) \mathbf{k}$ (i.e., fixed $\stackrel{\circ}{)}$ ) has tangent vector $\mathbf{t}=r^{\prime}(s) \mathbf{e}_{1}(\stackrel{o}{v})+z^{\prime}(s) \mathbf{k}$, where the primes denote differentiation with respect to $s$. Because we parametrize the meridian by arclength, the tangent vector has unit length, i.e., $r^{\prime}(s)^{2}+z^{\prime}(s)^{2}=1$. Hence, we can define

$$
r^{\prime}(s)=-\sin \theta(s), \quad z^{\prime}(s)=\cos \theta(s)
$$

where $\theta(s)$ is the angle between $\mathbf{t}$ and $\mathbf{k}$, and write $\mathbf{t}=-\sin \theta \mathbf{e}_{1}+\cos \theta \mathbf{k}$. Note that, since we assume $z$ increases with $s$, $\mathbf{t}$ has an upward component. Hence, the angle $\theta$ is positive to the "left" of $\mathbf{k}$ in the plane of the profile curve.

There is also a unit normal $\mathbf{n}(s, v)$ to the surface $\mathbf{x}(s, v)$ determined as follows: the unit vector $\mathbf{t}$ and the vector $\mathbf{x}_{v}=r(s) \mathbf{e}_{2}$ give a basis for the tangent plane to $\mathbf{x}(s, v)$, so

$$
\mathbf{n}(s, v)=\frac{\mathbf{t} \times \mathbf{x}_{v}}{\left|\mathbf{t} \times \mathbf{x}_{v}\right|}=-\cos \theta(s) \mathbf{e}_{1}(v)-\sin \theta(s) \mathbf{k}
$$

is the desired unit normal. For a surface of revolution parameterized in the form $(h(u) \cos v, h(u) \sin v, g(u))$ with unit normal as given above, we know (see [22, 
Section 3.3.3]) that the principal curvatures are given by

$$
k_{\mu}=\frac{g^{\prime \prime} h^{\prime}-g^{\prime} h^{\prime \prime}}{\left(g^{\prime 2}+h^{\prime 2}\right)^{3 / 2}}, \quad k_{\pi}=\frac{g^{\prime}}{h\left(g^{2}+h^{\prime 2}\right)^{1 / 2}} .
$$

The subscript $\mu$ denotes that $k_{\mu}$ is the curvature of the meridian (given by the intersection of the plane determined by $\mathbf{t}$ and $\mathbf{n}$ at any point and $\mathbf{x}$ ). The subscript $\pi$ denotes that $k_{\pi}$ is the curvature given by the intersection of the plane determined by $\mathbf{x}_{v}$ and $\mathbf{n}$ at any point and $\mathbf{x}$. For our surface $\mathbf{x}(s, v)$, we have $g=z$ and $h=r$, so we obtain

$$
\begin{aligned}
k_{\mu} & =\frac{z^{\prime \prime} r^{\prime}-z^{\prime} r^{\prime \prime}}{1}=\left(\frac{-r^{\prime} r^{\prime \prime}}{z^{\prime}}\right) r^{\prime}-z^{\prime} r^{\prime \prime} \\
& =\frac{-r^{\prime \prime}}{z^{\prime}}=\frac{\cos \theta \theta^{\prime}}{\cos \theta}=\theta^{\prime}
\end{aligned}
$$

where we have used $r^{\prime}(s)^{2}+z^{\prime}(s)^{2}=1$ and $r^{\prime} r^{\prime \prime}+z^{\prime} z^{\prime \prime}=0$ (by differentiating the first equation). We also obtain

$$
k_{\pi}=\frac{z^{\prime}}{r}=\frac{\cos \theta}{r} .
$$

These principal curvatures will help us understand crucial interactions between tensions and geometry later.

\subsection{Tensions}

Curved membranes do not necessarily change geometry to resist smoothly distributed loads. Furthermore, because shapes are determined at equilibrium, stresses may be found by solving differential equations (with boundary conditions). For arguments justifying these conclusions, see [1] and [13, Section 5.1]. So our goal is to find the determining differential equations.

There are three possible tensions to consider: the meridian stress $\sigma_{m}$ in the direction $\mathbf{t}$, the circumferential (or hoop) stress $\sigma_{c}$ in the direction $\mathbf{e}_{2}$ and the shear stress. As is argued in [13], for membranes that are surfaces of revolution, shear stresses are zero due essentially to symmetry about an axis. These internal tensions are given in units of force per unit length. An inflated membrane has an external pressure $p(s) \overline{\mathbf{n}}(s, v)-w(s) \mathbf{k}$, where $\overline{\mathbf{n}}=-\mathbf{n}$ is the outward normal, the pressure $p(s)$ depends only on the meridian parameter $s$ by symmetry about the $z$-axis and $w(s)$ is a weight density associated to the membrane itself. Note that pressure pushes the membrane outward normally while weight is directed downward as usual. Consider a patch on the membrane (see Fig. 1) with parameter 


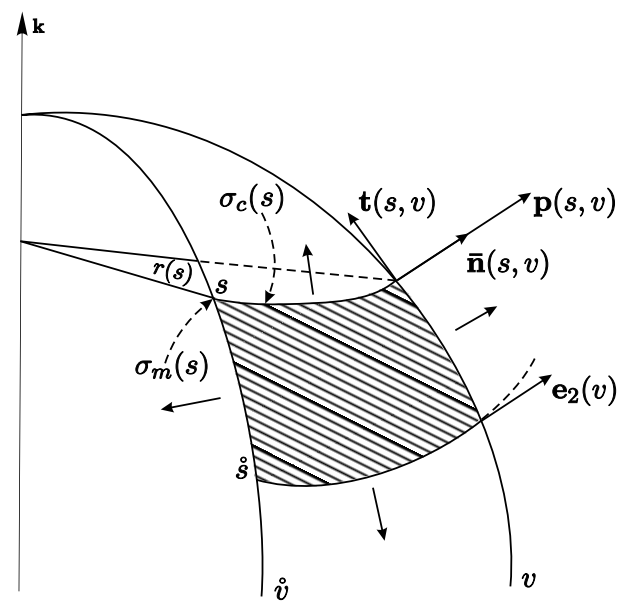

Figure 1: A patch on an axisymmetric membrane which is in equilibrium under various forces acting on it.

bounds $\stackrel{s}{s} s$ and $\dot{v} \leq v$. The patch is in equilibrium, so the total force acting on it is zero. Instead of writing things componentwise, we use vector notation and write

$$
\begin{aligned}
& 0=\int_{\dot{v}}^{v} \sigma_{m}(s) r(s) \mathbf{t}(s, u) \mathrm{d} u-\int_{\dot{v}}^{v} \sigma_{m}(\stackrel{\circ}{s}) r(\stackrel{\circ}{s}) \mathbf{t}(\stackrel{\circ}{s}, u) \mathrm{d} u \\
& +\int_{\dot{s}}^{s} \sigma_{c}(t) \mathbf{e}_{2}(v) \mathrm{d} t-\int_{\dot{s}}^{s} \sigma_{c}(t) \mathbf{e}_{2}(\grave{v}) \mathrm{d} t \\
& +\int_{\check{s}}^{s} \int_{\grave{v}}^{v} p(t) r(t) \overline{\mathbf{n}}(t, u) \mathrm{d} u \mathrm{~d} t-\int_{\grave{s}}^{s} \int_{\dot{v}}^{v} w(t) r(t) \mathbf{k} \mathrm{d} u \mathrm{~d} t .
\end{aligned}
$$

Now take $\partial / \partial s$ on both sides to obtain:

$$
\begin{aligned}
0= & \int_{\dot{v}}^{v} \frac{\partial}{\partial s}\left(\sigma_{m}(s) r(s) \mathbf{t}(s, u)\right) \mathrm{d} u+\sigma_{c}(s) \mathbf{e}_{2}(v)-\sigma_{c}(s) \mathbf{e}_{2}(\stackrel{\vartheta}{v}) \\
& +\int_{\grave{v}}^{v} p(s) r(s) \overline{\mathbf{n}}(s, u) \mathrm{d} u-\int_{\grave{v}}^{v} w(s) r(s) \mathbf{k} \mathrm{d} u .
\end{aligned}
$$

Take $\partial / \partial v$ on both sides of this equation to obtain:

$$
0=\frac{\partial}{\partial s}\left(\sigma_{m}(s) r(s) \mathbf{t}(s, v)\right)-\sigma_{c}(s) \mathbf{e}_{1}(v)+p(s) r(s) \overline{\mathbf{n}}(s, v)-w(s) r(s) \mathbf{k} .
$$

Now we can project onto $\mathbf{t}$ and $\overline{\mathbf{n}}$ by dotting with $\mathbf{t}$ and $\overline{\mathbf{n}}$ respectively. We use 
several facts: $\mathbf{t} \cdot \partial \mathbf{t} / \partial s=0, \mathbf{e}_{1} \cdot \mathbf{t}=-\sin \theta$ and

$$
\begin{aligned}
\frac{\partial}{\partial s}\left(\sigma_{m} r \mathbf{t}\right) \cdot \mathbf{t}+\left(\sigma_{m} r \mathbf{t}\right) \cdot \frac{\partial \mathbf{t}}{\partial s} & =\frac{\partial}{\partial s}\left(\sigma_{m} r \mathbf{t} \cdot \mathbf{t}\right) \\
& =\frac{\partial}{\partial s}\left(\sigma_{m} r\right)+2\left(\sigma_{m} r\right) \frac{\partial \mathbf{t}}{\partial s} \cdot \mathbf{t} \\
\frac{\partial}{\partial s}\left(\sigma_{m} r \mathbf{t}\right) \cdot \mathbf{t} & =\frac{\partial}{\partial s}\left(\sigma_{m} r\right) .
\end{aligned}
$$

We therefore have

$$
\begin{aligned}
0 & =\frac{\partial}{\partial s}\left(\sigma_{m} r\right)+\sigma_{c} \sin \theta-w r \cos \theta \\
\frac{\partial}{\partial s}\left(\sigma_{m} r\right) & =-\sigma_{c} \sin \theta+w r \cos \theta
\end{aligned}
$$

Dotting with $\overline{\mathbf{n}}$ gives (using $\partial \mathbf{t} / \partial s=-\theta^{\prime} \overline{\mathbf{n}}$ )

$$
\begin{aligned}
0 & =\frac{\partial}{\partial s}\left(\sigma_{m} r \mathbf{t}\right) \cdot \overline{\mathbf{n}}-\sigma_{c} \mathbf{e}_{1} \cdot \overline{\mathbf{n}}+p r \overline{\mathbf{n}} \cdot \overline{\mathbf{n}}-w r \mathbf{k} \cdot \overline{\mathbf{n}} \\
0 & =-\sigma_{m} r \theta^{\prime}-\sigma_{c} \cos \theta+p r-w r \sin \theta \\
\sigma_{m} r \theta^{\prime} & =p r-\sigma_{c} \cos \theta-w r \sin \theta .
\end{aligned}
$$

\subsection{The case $w=0$}

Let's consider the case where the weight of the membrane is negligible; that is, $w=0$. Recall that $k_{\mu}=\theta^{\prime}$ and $k_{\pi}=\cos \theta / r$. From (5), we get

$$
\begin{aligned}
\sigma_{m} \theta^{\prime}+\frac{\sigma_{c}}{r} \cos \theta & =p \\
\sigma_{m} k_{\mu}+\sigma_{c} k_{\pi} & =p .
\end{aligned}
$$

If we define the radii of curvature by $r_{\mu}=1 / k_{\mu}$ and $r_{\pi}=1 / k_{\pi}$, then we have a version of the Laplace-Young equation (see [21,22] for instance):

$$
\frac{\sigma_{m}}{r_{\mu}}+\frac{\sigma_{c}}{r_{\pi}}=p .
$$

Remark 1. Of course, when $w \neq 0$, we then have

$$
\frac{\sigma_{m}}{r_{\mu}}+\frac{\sigma_{c}}{r_{\pi}}=p-\frac{w}{r} \sin \theta
$$


Now, when $w=0$, (4) becomes $\frac{\partial}{\partial s}\left(\sigma_{m} r\right)=-\sigma_{c} \sin \theta$. Since $r^{\prime}=-\sin \theta$, a solution is given by

$$
\sigma_{m}=\sigma_{c}=\sigma, \quad \text { a constant. }
$$

Put this in the Laplace-Young equation (6) to get

$$
\begin{aligned}
\sigma\left(\theta^{\prime}+\frac{\cos \theta}{r}\right) & =p \\
\frac{1}{2}\left(k_{\mu}+k_{\pi}\right) & =\frac{1}{2} \frac{p}{\sigma} \\
H & =\frac{1}{2} \frac{p}{\sigma}
\end{aligned}
$$

where $H$ is the mean curvature of the membrane.

If the pressure $p$ is constant, then $H$ is constant as well and the membrane is a surface of Delaunay (see [21,22]).

It is easy to conclude also that when $p$ is still constant, but $\sigma_{m} \neq \sigma_{c}$ the equation (6) defines the so called anisotropic Delaunay surfaces, and the reader is referred for more details on their subject to [16].

Finally, if $p=0$ and $\sigma_{m} \neq \sigma_{c}$, one ends with the quite interesting class of the linear Weingarten surfaces $[18,20]$.

Consider (4) again (when $w=0$ ) and suppose $\sigma_{c}=0, p=\alpha$, constant. Then $\sigma_{m} r=\beta$ is a constant and $\sigma_{m}=\beta / r$. From (5), we get (using $r^{\prime}=-\sin \theta$ )

$$
\begin{aligned}
\frac{\beta}{r} r \theta^{\prime} & =\alpha r \\
\beta \theta^{\prime} & =\alpha r \\
\theta^{\prime} & =\frac{\alpha}{\beta} r \\
2 r^{\prime} \theta^{\prime} & =2 \frac{\alpha}{\beta} r r^{\prime} \\
-2 \sin \theta \theta^{\prime} & =2 \frac{\alpha}{\beta} r r^{\prime} \\
2(\cos \theta)^{\prime} & =\frac{\alpha}{\beta}\left(r^{2}\right)^{\prime} \\
2 \cos \theta & =\frac{\alpha}{\beta} r^{2}+d .
\end{aligned}
$$

From our assumptions that the profile curve rises from the $x$-axis and goes to the $z$-axis orthogonally, we see that $\theta=\pi / 2$ exactly when $r=0$. Hence, $d=0$. Therefore, we have 


$$
\begin{aligned}
2 \frac{\cos \theta}{r} & =\frac{\alpha}{\beta} r \\
& =\theta^{\prime} \\
2 k_{\pi} & =k_{\mu} .
\end{aligned}
$$

This condition will be explored in the next section. It describes the Mylar balloon. We can thus say the following.

Theorem 2. If a membrane with $w=0$, constant pressure $p$ and hoop stress $\sigma_{c}=0$ is a surface of revolution

$$
\mathbf{x}(s, v)=(r(s) \cos v, r(s) \sin v, z(s))=r(s) \mathbf{e}_{1}(v)+z(s) \mathbf{e}_{3}(v)
$$

then $2 k_{\pi}=k_{\mu}$; that is, the membrane is a Mylar balloon (see Section 3).

\section{The Mylar Balloon}

Let's start with a mathematical model of a Mylar balloon. A Mylar balloon is constructed from two disks of Mylar (of radius $a$ say) sewn along their edges. When the Mylar disk is inflated, the radius deforms to a curve $z=z(x)$ that we take to be in the first quadrant of the $x z$-plane. Of course, physical intuition implies that the curve proceeds from its highest point on the $z$-axis downward to a point of intersection with the $x$-axis (with respect to the parameter $x$, not $s$ as in Subsection 2.1). This is the right-hand side of the curve that, when revolved about the $z$-axis, produces the top half of the balloon. The bottom half is just a reflection of the upper half in the $x y$-plane.

Let $R$ be the radius of the inflated balloon (i.e., the distance along the $x$-axis from $x=0$ to the point of intersection with the $x$-axis). Because of its physical properties, the Mylar does not stretch significantly. As a result, the arclength of the graph of $z(x)$ from $x=0$ to $x=R$ is equal to the initial radius $a$. That is, we have

$$
\int_{0}^{R} \sqrt{1+z^{\prime}(x)^{2}} \mathrm{~d} x=a .
$$

The basic shape of the balloon is determined by this constraint. Next, it is clear that, when the balloon is inflated, the pressure of the gas inside induces a maximal enclosed volume. Because the balloon is a surface of revolution, this volume is 
given by the shell method:

$$
V=4 \pi \int_{0}^{R} x z(x) \mathrm{d} x .
$$

In this setting we now have the calculus of variations problem of extremizing $V$ subject to the constraint (9). The corresponding Euler-Lagrange equation is

$$
\frac{\mathrm{d}}{\mathrm{d} x}\left(\frac{\lambda z^{\prime}(x)}{\sqrt{1+z^{\prime}(x)^{2}}}\right)-4 \pi x=0 .
$$

This equation is easy to integrate, yielding

$$
\frac{\lambda z^{\prime}(x)}{\sqrt{1+z^{\prime}(x)^{2}}}=2 \pi x^{2}+C
$$

for some constant of integration $C$. This constant can be found by taking into account the obvious geometrical (transversality) condition $z^{\prime}(0)=0$. Inserting $x=0$ into (11) and using $z^{\prime}(0)=0$ gives $C=0$. Consequently, we obtain

$$
\frac{z^{\prime}(x)}{\sqrt{1+z^{\prime}(x)^{2}}}=\frac{2 \pi}{\lambda} x^{2} .
$$

The transversality condition $\lim _{x \rightarrow R^{-}} z^{\prime}(x)=-\infty$, along with the requirement that the curve proceed from its highest point to its intersection with the $x$-axis without introducing critical points (i.e., $z^{\prime}(x)<0$ when $0<x<R$ ), allows us to rewrite $\lambda /(2 \pi)$ in the form $-m^{2}$. Now, solving (12) for $z^{\prime}(x)$, we find that

$$
z^{\prime}(x)=-\frac{x^{2}}{\sqrt{m^{4}-x^{4}}}
$$

and, integrating, that

$$
z(x)=\int_{x}^{R} \frac{t^{2}}{\sqrt{m^{4}-t^{4}}} \mathrm{~d} t
$$

where the choice of the upper limit of integration comes from the requirement that $z(R)=0$. The profile curve of the Mylar balloon will be completely determined if we know what $m$ is. Its value can be ascertained if we remember that $\lim _{x \rightarrow R^{-}} z^{\prime}(x)=-\infty$ and note that this will be the case if we take $m=R$. Therefore, in the end we have

$$
z(x)=\int_{x}^{R} \frac{t^{2}}{\sqrt{R^{4}-t^{4}}} \mathrm{~d} t, \quad 0 \leq x \leq R .
$$


This is an elliptic integral that has no closed form solution in terms of "elementary" functions. In what follows, however, we find an explicit expression for the integral (13) in terms of elliptic functions and then use this expression to derive various facts associated with the geometry of the Mylar balloon. For that purpose we change the integration variable from $t$ to $u$ and make the substitution $t=R \operatorname{cn}(u, k)$ where $\operatorname{cn}(u, k)$ is the Jacobi cosine function. The basic properties of elliptic functions (details about which can be found in [14]) and this substitution reduce (13) to

$$
z(u)=\frac{R}{\sqrt{2}} \int_{0}^{u_{0}} \frac{\mathrm{cn}^{2}(u, k) \mathrm{dn}(u, k) \mathrm{d} u}{\sqrt{1-\frac{1}{2} \operatorname{sn}^{2}(u, k)}} .
$$

Here $u_{0}$ satisfies $\operatorname{cn}\left(u_{0}, k\right)=x / R$. Also, we use the identity

$$
1-\operatorname{cn}^{4}(u, k)=\left(1-\operatorname{cn}^{2}(u, k)\right)\left(1+\operatorname{cn}^{2}(u, k)\right)=\operatorname{sn}^{2}(u, k)\left(2-\operatorname{sn}^{2}(u, k)\right) .
$$

Further simplification of (14) can be achieved by choosing the value of the elliptic modulus $k$ appropriately. Letting $k=1 / \sqrt{2}$, we note that the identity $\operatorname{dn}^{2}(u, k)+$ $k^{2} \operatorname{sn}^{2}(u, k)=1$ gives

$$
z(u)=\frac{R}{\sqrt{2}} \int_{0}^{u} \operatorname{cn}^{2}\left(\tilde{u}, \frac{1}{\sqrt{2}}\right) \mathrm{d} \tilde{u} .
$$

We now have the following general result (see [19] and also [7, item 782.02]).

Proposition 3. The following identity holds for $u>0:{ }^{1}$

$$
\int_{0}^{u} \operatorname{cn}^{2}(\tilde{u}, k) \mathrm{d} \tilde{u}=\frac{E(\operatorname{sn}(u, k), k)}{k^{2}}-\frac{1-k^{2}}{k^{2}} F(\operatorname{sn}(u, k), k) .
$$

Here $F(\phi, k)$ and $E(\phi, k)$ are the so called incomplete elliptic integrals of the first, respectively second kind which are functions of their argument $\phi$ and the parameter $k$ that is the elliptic modulus mentioned earlier. We can then express $z(u)$ in terms of elliptic functions:

$$
z(u)=R \sqrt{2}\left[E\left(\operatorname{sn}\left(u, \frac{1}{\sqrt{2}}\right), \frac{1}{\sqrt{2}}\right)-\frac{1}{2} F\left(\operatorname{sn}\left(u, \frac{1}{\sqrt{2}}\right), \frac{1}{\sqrt{2}}\right)\right] .
$$

Since $t=R \operatorname{cn}(u, k)$, this means that the profile curve (traced counterclockwise) is given by

\footnotetext{
${ }^{1}$ Although $F\left(\operatorname{sn}\left(u_{0}, k\right), k\right)=u_{0}$, we shall continue to write $F\left(\operatorname{sn}\left(u_{0}, k\right), k\right)$ for uniformity.
} 

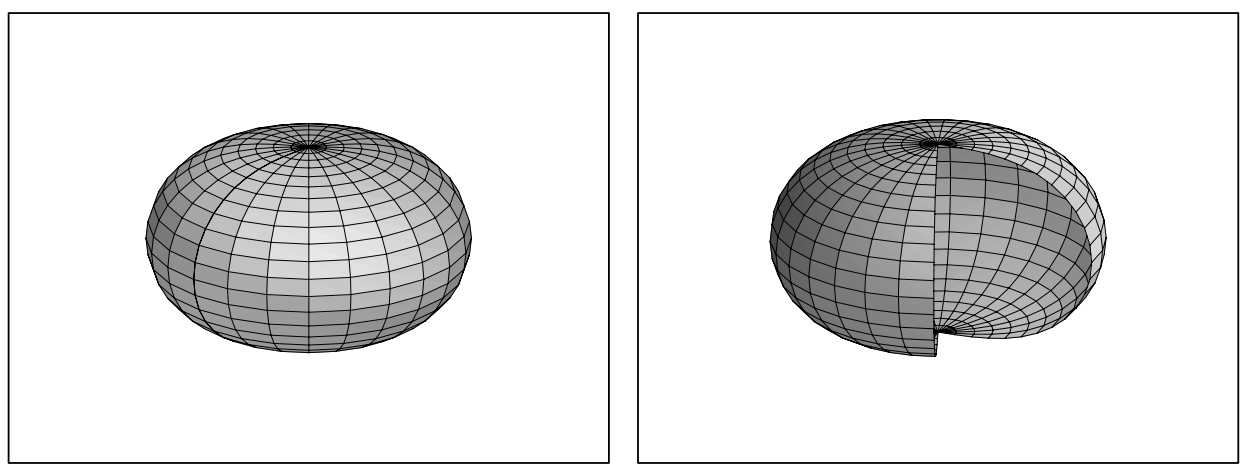

Figure 2: Two views of the Mylar balloon.

$$
\begin{aligned}
& x(u)=R \operatorname{cn}\left(u, \frac{1}{\sqrt{2}}\right) \\
& z(u)=R \sqrt{2}\left[E\left(\operatorname{sn}\left(u, \frac{1}{\sqrt{2}}\right), \frac{1}{\sqrt{2}}\right)-\frac{1}{2} F\left(\operatorname{sn}\left(u, \frac{1}{\sqrt{2}}\right), \frac{1}{\sqrt{2}}\right)\right]
\end{aligned}
$$

for $u$ in $[0, K(1 / \sqrt{2})]$. Note that the complete integral $K(1 / \sqrt{2})$ arises because $\operatorname{cn}(u, 1 / \sqrt{2})$ varies from 1 (where $u=0$ ) to 0 (where $u=K(1 / \sqrt{2})$ ).

Theorem 4. The surface of revolution $\mathcal{S}$ that models the Mylar balloon is parametrized by

$$
\mathbf{x}=\mathbf{x}(u, v)=(x(u, v), y(u, v), z(u, v))
$$

where for $u$ in $[-K(1 / \sqrt{2}), K(1 / \sqrt{2})]$ and $v$ in $[0,2 \pi]$

$$
\begin{aligned}
& x(u, v)=R \operatorname{cn}\left(u, \frac{1}{\sqrt{2}}\right) \cos v, \quad y(u, v)=R \operatorname{cn}\left(u, \frac{1}{\sqrt{2}}\right) \sin v \\
& z(u, v)=R \sqrt{2}\left[E\left(\operatorname{sn}\left(u, \frac{1}{\sqrt{2}}\right), \frac{1}{\sqrt{2}}\right)-\frac{1}{2} F\left(\operatorname{sn}\left(u, \frac{1}{\sqrt{2}}\right), \frac{1}{\sqrt{2}}\right)\right] .
\end{aligned}
$$

We can now put this parametrization into a computer algebra system such as Maple and plot. We then see the familiar shape of a Mylar balloon in Fig. 2. 
Theorem 5. A surface of revolution $M$ given by

$$
\mathbf{x}(u, v)=(r(u) \cos v, r(u) \sin v, z(u))
$$

such that $k_{\mu}=2 k_{\pi}$ has a parametrization of the form (17). That is, the surface $M$ is a Mylar balloon.

Proof: By reparametrizing, we may without loss of generality assume that the profile curve $(h(u), g(u))$ of $M$ has constant speed. (Note that the parametrization of the Mylar balloon that we have given satisfies this without reparametrization: $\sqrt{x^{\prime}(u)^{2}+z^{\prime}(u)^{2}}=R / \sqrt{2}$. $)^{2}$ We thus have $g^{\prime}(u)^{2}+h^{\prime}(u)^{2}=A^{2}$ for some constant $A>0$. By differentiating this relation with respect to $u$, we find that $g^{\prime} g^{\prime \prime}+h^{\prime} h^{\prime \prime}=0$. In tandem, these two relations reduce the principal curvature formulas (see (2)) to

$$
k_{\mu}=\frac{g^{\prime \prime}}{A h^{\prime}} \quad k_{\pi}=\frac{g^{\prime}}{A h} .
$$

The hypothesis $k_{\mu}=2 k_{\pi}$ then gives

$$
\frac{g^{\prime \prime}}{g^{\prime}}=2 \frac{h^{\prime}}{h}
$$

which ensures that $g^{\prime}=\alpha h^{2}$ for some constant $\alpha$. We now insert this into the constant speed relation to obtain $\mathrm{d} h / \mathrm{d} u=h^{\prime}= \pm \sqrt{A^{2}-\alpha^{2} h^{4}}$. This is a separable differential equation that can be solved by making the substitutions $h=\sqrt{A / \alpha} \mathrm{cn} w, \mathrm{~d} h=-\sqrt{A / \alpha} \operatorname{sn} w \operatorname{dn} w \mathrm{~d} w$ (where the elliptic modulus is always taken to be $1 / \sqrt{2})$ and using the identity $1-\operatorname{cn}^{4} w=\operatorname{sn}^{2} w\left(2-\operatorname{sn}^{2} w\right)$ :

$$
\begin{gathered}
\pm A \int \mathrm{d} u=\int \frac{\mathrm{d} h}{\sqrt{1-\left(\frac{\alpha}{A}\right)^{2} h^{4}}} \\
\pm A u+c=\int \frac{\sqrt{\frac{A}{\alpha}} \operatorname{sn} w \mathrm{dn} w}{\sqrt{2} \operatorname{sn} w \sqrt{1-\frac{1}{2} \operatorname{sn}^{2} w}} \mathrm{~d} w=\sqrt{\frac{A}{2 \alpha}} \int \mathrm{d} w=\sqrt{\frac{A}{2 \alpha}} w
\end{gathered}
$$

whence $\sqrt{2 \alpha A} u+\bar{c}=w$, where $\bar{c}$ is a constant. We then apply the elliptic cosine and use the substitution relation to obtain

$$
\sqrt{\frac{\alpha}{A}} h=\operatorname{cn}(\sqrt{2 \alpha A} u+\bar{c})
$$

\footnotetext{
${ }^{2}$ Note that an extraneous $K$ appears here as a result of a TeX copy-paste error in [19, Theorem 6.5] and [22, Theorem 7.9.4] where the square root is said to be $R K / \sqrt{2}$.
} 


$$
h(u)=\sqrt{\frac{A}{\alpha}} \operatorname{cn}(\sqrt{2 \alpha A} u+\bar{c}) .
$$

We take $\bar{c}=0$ for convenience and find $g(u)$ by recalling that $g^{\prime}=\alpha h^{2}=$ $A \mathrm{cn}^{2}(\sqrt{2 \alpha A} u)$ and invoking Proposition 3:

$$
\begin{aligned}
g(u) & =A \int \operatorname{cn}^{2}(\sqrt{2 \alpha A} u) \mathrm{d} u \\
& =\frac{A}{\sqrt{2 \alpha A}}\left(2 E\left(\operatorname{sn}(u), \frac{1}{\sqrt{2}}\right)-F\left(\operatorname{sn}(u), \frac{1}{\sqrt{2}}\right)\right) \\
& =\sqrt{\frac{2 A}{\alpha}}\left(E\left(\operatorname{sn}(u), \frac{1}{\sqrt{2}}\right)-\frac{1}{2} F\left(\operatorname{sn}(u), \frac{1}{\sqrt{2}}\right)\right) .
\end{aligned}
$$

This results in a parametrization of type (17). (Note that, for the balloon in (17), $A=R / \sqrt{2}$ and $\alpha=1 /(\sqrt{2} R)$.)

Remark 6. The natural parametrization of the balloon profile curve (16) can be obtained after replacement of the argument $u$ there with $\frac{\sqrt{2} s}{R}$. We can also easily find that its curvature is then given by

$$
\kappa(s)=k_{\mu}(s)=\frac{2}{R} \operatorname{cn}\left(\frac{\sqrt{2} s}{R}, \frac{1}{\sqrt{2}}\right)
$$

and that it satisfies the fundamental equation of the Euler elastica without a length constraint (i.e., the so called free elastica)

$$
\ddot{\kappa}(s)+\frac{1}{2} \kappa^{3}(s)=0
$$

found by Birkhoff and de Boor [6]. (Here, the dots denote the derivatives with respect to the natural parameter s.)

\section{Kawaguchi's Domes}

\subsection{The Question of the Shallowest Dome}

We have seen in Theorem 5 that the Mylar balloon is characterized by being a surface of revolution with $k_{\mu}=2 k_{\pi}$. Also, in Theorem 2, we then saw that a weightless membrane with no hoop stress is a Mylar balloon. Amazingly, there is 


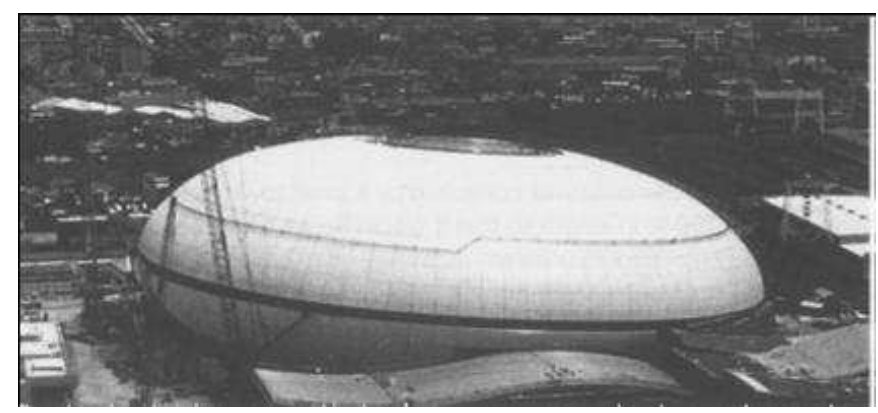

Figure 3: The Namihaya Sports Hall Dome.

yet another criterion that produces the Mylar balloon and this criterion was known years ago in the world of structural engineering. In [15] (also see [13, Example 5.2]), the structural engineer Mamoru Kawaguchi asked the following

Question 7. What shape is the pneumatic dome which has smallest height to span ratio, meets the ground at a right angle and no wrinkles?

A pneumatic dome is a membrane-like structure which is supported by internal air pressure slightly higher than normal atmospheric pressure. Such a dome is not a membrane precisely, but it is common to use this approximation as an ideal model. One famous approach to constructing these domes is called the Pantadome system. This approach allows supporting members in the hoop direction to be removed in order to fold the membrane for transport. Then the membrane is inflated and, once at the desired volume, the supporting members are reinserted. Kawaguchi's Namihaya Sports Hall Dome is an example (see Fig. 3).

In order to answer Question 7, Kawaguchi assumes that the pneumatic dome is "hemispherical"; that is, it is a surface of revolution about the $z$-axis with generating meridian meeting the $x$-axis orthogonally and increasing in $z$ until it meets the $z$-axis orthogonally. Of course, this is the type of surface of revolution we have been dealing with in Section 2 and Section 3.

First, note that the Laplace-Young formula (6) holds:

$$
\frac{\sigma_{m}}{r_{\mu}}+\frac{\sigma_{c}}{r_{\pi}}=p
$$

where we assume the weight of the dome material is negligible. Now slice the dome horizontally by a plane to obtain a disk of radius $r$. The pressure $p$ acts upward on the disk with force $F=p \pi r^{2}$, but since the membrane supports this 
(imaginary) disk, we can uniformly distribute $F$ over the circumference of the disk to obtain a stress $\sigma_{d}=\left(p \pi r^{2}\right) /(2 \pi r)=p r / 2 .^{3}$

Now, $\sigma_{m}$ acts along $\mathbf{t}$ and $\sigma_{d}$ acts in the k-direction, so to balance forces, we need

$$
\sigma_{m} \cos \theta=\sigma_{d}=\frac{p r}{2}
$$

or $\sigma_{m}=p /\left(2 k_{\pi}\right)$. Substituting into Laplace-Young then gives

$$
\sigma_{c}=\frac{p}{k_{\pi}}\left(1-\frac{k_{\mu}}{2 k_{\pi}}\right) \text {. }
$$

Kawaguchi notes that, for a pneumatic dome with no wrinkles, it must be the case that $\sigma_{m} \geq 0$ and $\sigma_{c} \geq 0$. Therefore, it is necessary to have $k_{\pi} \geq 0$ and $k_{\mu} \leq 2 k_{\pi}$. In order to answer Question 7, it is easier to use $r_{\pi}=1 / k_{\pi}$ and $r_{\mu}=1 / k_{\mu}$. The conditions then become $r_{\pi} \geq 0$ and $r_{\mu} \geq r_{\pi} / 2$. These conditions then say that there is a function $f(\theta) \geq 0$ with $r_{\mu}=r_{\pi} / 2+f(\theta)$.

For the shallowest dome, we must minimize $h / R$ where $h=z\left(s_{1}\right)$ is the height of the dome (i.e., the $z$-coordinate of the intersection of the meridian with the $z$-axis). We then can calculate as follows.

$$
\begin{aligned}
\frac{h}{R} & =\frac{1}{R} \int_{0}^{s_{1}} z^{\prime}(s) \mathrm{d} s=\int_{0}^{\pi / 2} z^{\prime}(s) \frac{\mathrm{d} s}{\mathrm{~d} \theta} \mathrm{d} \theta=\int_{0}^{\pi / 2} \frac{\cos \theta}{\theta^{\prime}} \mathrm{d} \theta \\
& =\frac{1}{R} \int_{0}^{\pi / 2} \frac{\cos \theta}{k_{\mu}} \mathrm{d} \theta=\frac{1}{R} \int_{0}^{\pi / 2} r_{\mu} \cos \theta \mathrm{d} \theta=\frac{1}{R} \int_{0}^{\pi / 2}\left(\frac{r_{\pi}}{2}+f\right) \cos \theta \mathrm{d} \theta \\
& =\frac{1}{R} \int_{0}^{\pi / 2} \frac{r_{\pi}}{2} \cos \theta \mathrm{d} \theta+\frac{1}{R} \int_{0}^{\pi / 2} f \cos \theta \mathrm{d} \theta .
\end{aligned}
$$

For $h / R$ to be a minimum, we therefore need $f=0$; that is, $r_{\mu}=r_{\pi} / 2$. Hence, we have

Theorem 8. The shallowest pneumatic dome has $k_{\mu}=2 k_{\pi}$. That is, it is a Mylar balloon.

Remark 9. Note that (18) implies that $\sigma_{c}=0$, so Kawaguchi's analysis correlates with Theorem 2.

\footnotetext{
${ }^{3}$ Thanks to Frank Baginski for explaining this argument to us.
} 


\subsection{Interpolating Between Sphere and Mylar Balloon}

Kawaguchi interpolates between the sphere (i.e., $k_{\mu}=k_{\pi}$ ) and the Mylar balloon (i.e., $k_{\mu}=2 k_{\pi}$ ). He supposes a relation

$$
\sigma_{c}=\sigma_{m} \mathrm{e}^{-\varepsilon \theta}
$$

Recalling $\sigma_{m}=p /\left(2 k_{\pi}\right)$ and $\sigma_{c}=\left(p / k_{\pi}\right)\left(1-k_{\mu} /\left(2 k_{\pi}\right)\right)$, we obtain

$$
k_{\pi}\left(2-\mathrm{e}^{-\varepsilon \theta}\right)=k_{\mu}
$$

Note that, when $\varepsilon=0$, then $k_{\mu}=k_{\pi}$ and we have a sphere (see [22, Theorem 3.5.2]). When $\varepsilon=\infty$, then $k_{\mu}=2 k_{\pi}$ and this is the Mylar balloon by Theorem 5 . The $\varepsilon$ 's between 0 and $\infty$ then give interesting new domes. For the parametrization

$$
\mathbf{x}(s, v)=(r(s) \cos v, r(s) \sin v, z(s))=r(s) \mathbf{e}_{1}(v)+z(s) \mathbf{e}_{3}(v)
$$

with $r^{\prime}(s)=-\sin \theta(s)$ and $z^{\prime}(s)=\cos \theta(s)$, where $\theta(s)$ is the angle between $\mathbf{t}$ and $\mathbf{k}$, we compute

$$
\begin{aligned}
k_{\mu} & =k_{\pi}\left(2-\mathrm{e}^{-\varepsilon \theta}\right) \\
\theta^{\prime} & =\frac{\cos \theta}{r}\left(2-\mathrm{e}^{-\varepsilon \theta}\right) \\
\frac{1}{\cos \theta\left(2-\mathrm{e}^{-\varepsilon \theta}\right)} \mathrm{d} \theta & =\frac{1}{r} \frac{\mathrm{d} s}{\mathrm{~d} r} \mathrm{~d} r \\
\frac{1}{\cos \theta\left(2-\mathrm{e}^{-\varepsilon \theta}\right)} \mathrm{d} \theta & =\frac{1}{r}\left(-\frac{1}{\sin \theta}\right) \mathrm{d} r \\
\frac{-\tan \theta}{\left(2-\mathrm{e}^{-\varepsilon \theta}\right)} \mathrm{d} \theta & =\frac{1}{r} \mathrm{~d} r \\
\int_{0}^{\theta} \frac{-\tan u}{\left(2-\mathrm{e}^{-\varepsilon u}\right)} \mathrm{d} u & =\int_{R}^{r} \frac{1}{r} \mathrm{~d} r=\ln \left(\frac{r}{R}\right) \\
r & =R \cdot \exp \left(-\int_{0}^{\theta} \frac{\tan u}{\left(2-\mathrm{e}^{-\varepsilon u}\right)} \mathrm{d} u\right) .
\end{aligned}
$$

We have thus obtained $r$, albeit defined using an integral. Now, $z^{\prime}=\cos \theta$, so the chain rule gives

$$
\frac{\mathrm{d} z}{\mathrm{~d} \theta}=\frac{\cos \theta}{\theta^{\prime}}
$$


Now, using (20) and the relations $k_{\mu}=\theta^{\prime}, k_{\pi}=\cos \theta / r$, we get

$$
\begin{aligned}
\frac{\mathrm{d} z}{\mathrm{~d} \theta} & =\frac{\cos \theta}{\cos \theta\left(2-\mathrm{e}^{-\varepsilon \theta}\right) / r}=\frac{r}{\left(2-\mathrm{e}^{-\varepsilon \theta}\right)} \\
& =R \frac{\exp \left(-\int_{0}^{\theta} \frac{\tan u}{\left(2-\mathrm{e}^{-\varepsilon u}\right)} \mathrm{d} u\right)}{\left(2-\mathrm{e}^{-\varepsilon \theta}\right)} .
\end{aligned}
$$

\subsection{The Interpolations and Maple}

We can use Maple to visualize the domes described by (21) and (23) which are presented in Figs. 4-9.

$>$ with(plots):with (DEtools) : with (LinearAlgebra) :

The following procedure pneumdome creates a profile curve for a pneumatic dome with radius $R$ and $\varepsilon=k$.

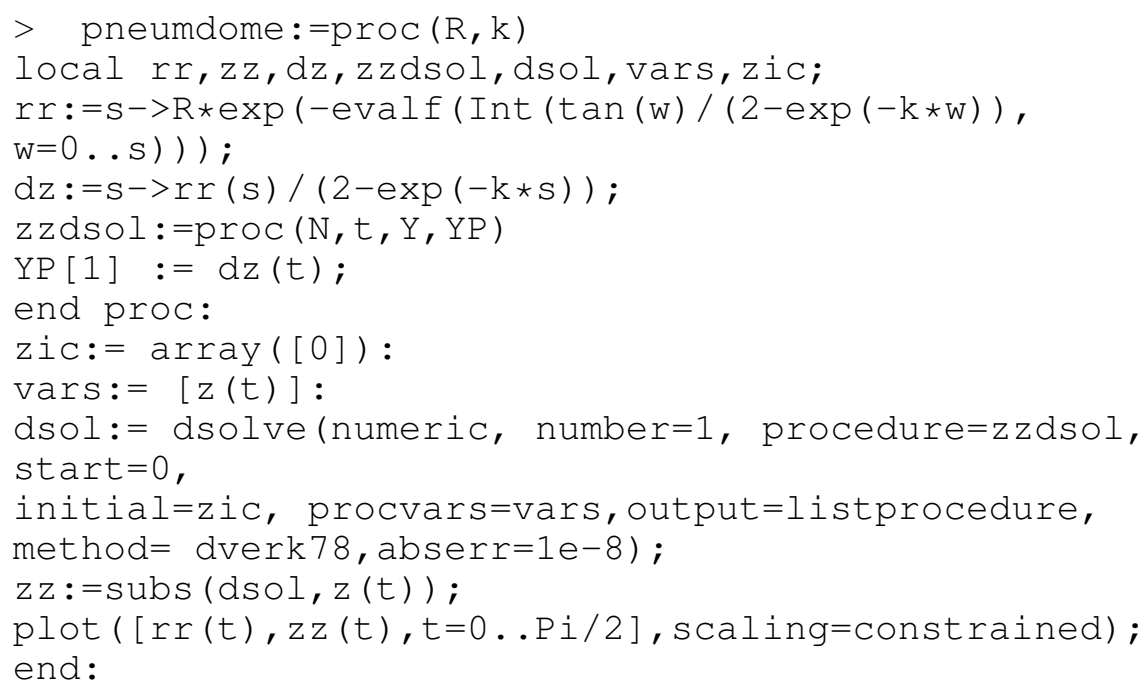

Now we can compute dome profile curves for many values of $\varepsilon$ and see the progression from sphere to Mylar balloon. Note that we cannot input $\infty$, so we must choose a large value for $\varepsilon$ to approximate it.

The following procedure pneumdomerev revolves profile curves about the $z$-axis to generate Kawaguchi domes.

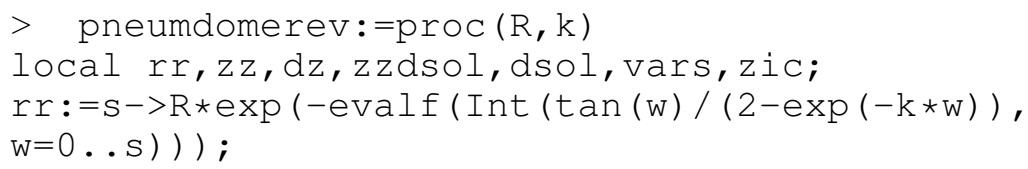




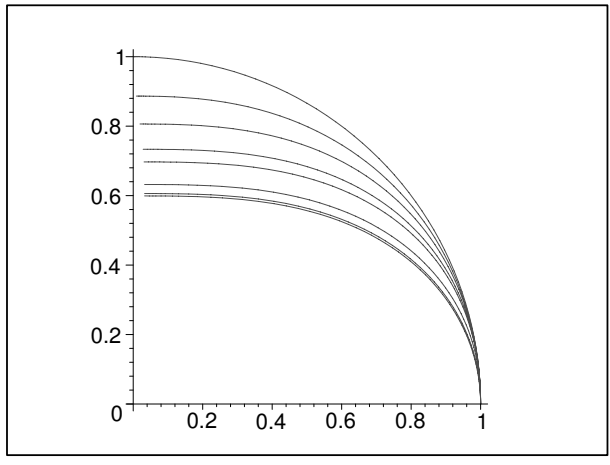

Figure 4: Dome profile curves.

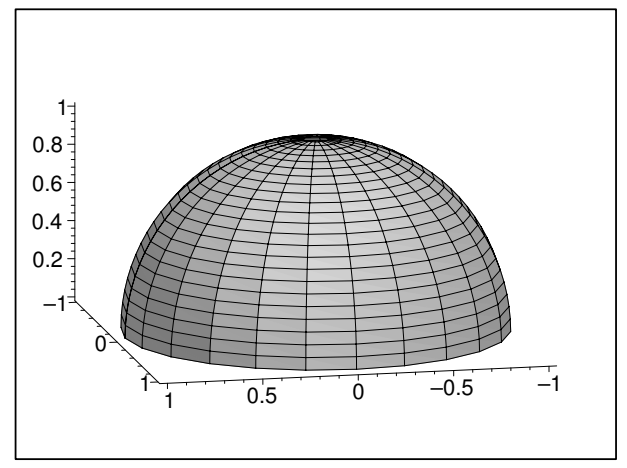

Figure 5: A spherical dome, $\varepsilon=0$.

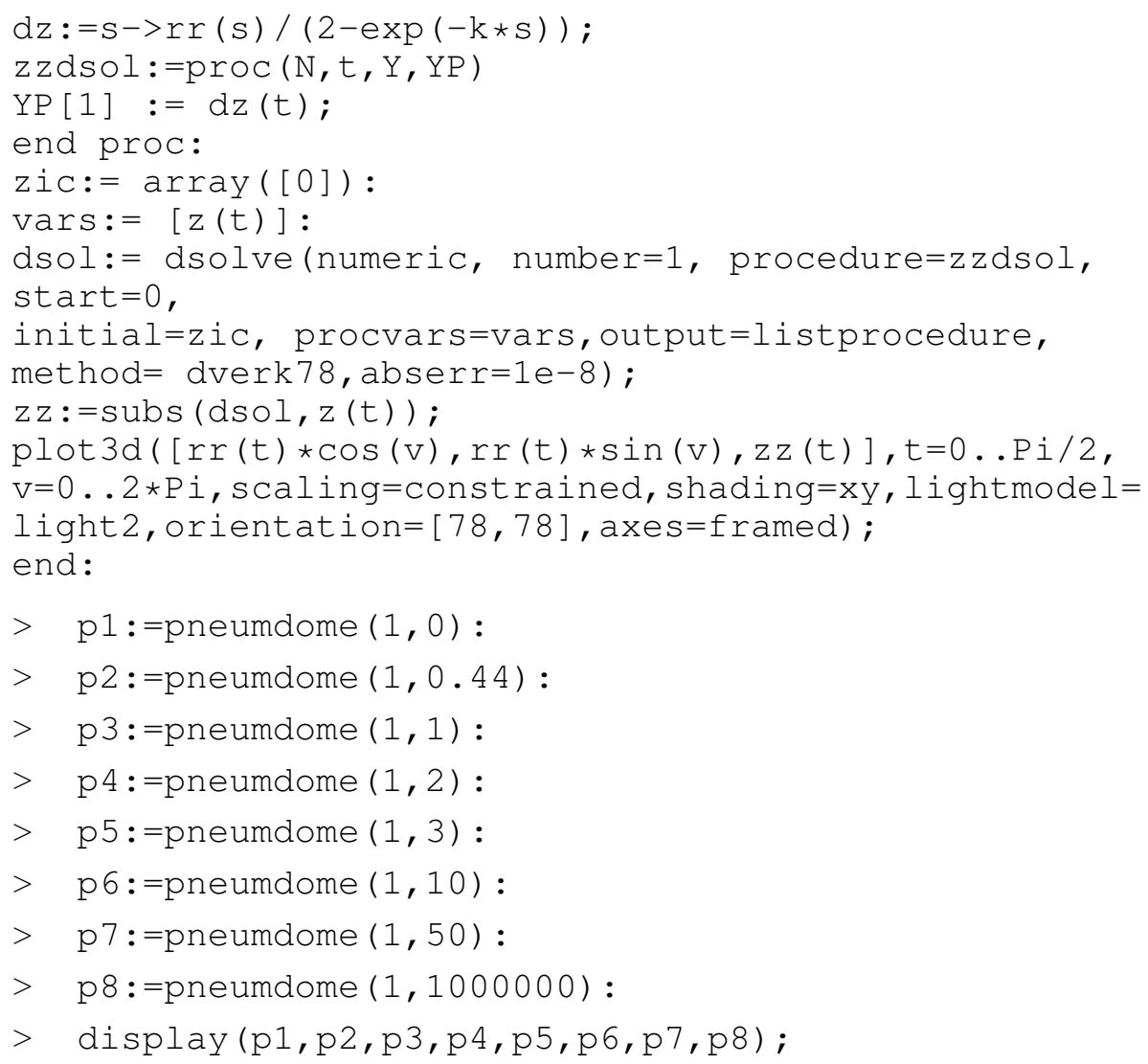

Here is the sphere. 


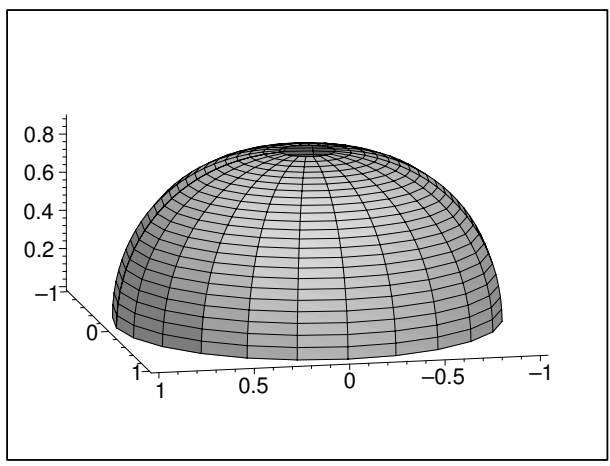

Figure 6: Almost spherical dome, $\varepsilon=0.44$.

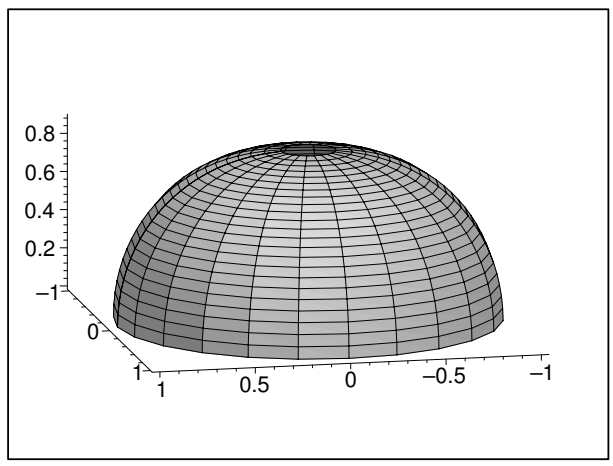

Figure 8: A dome approaching the Mylar dome, $\varepsilon=50$.

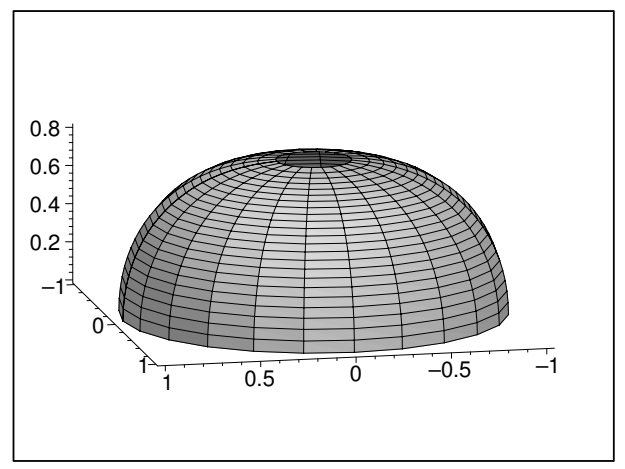

Figure 7: Another dome, $\varepsilon=1$.

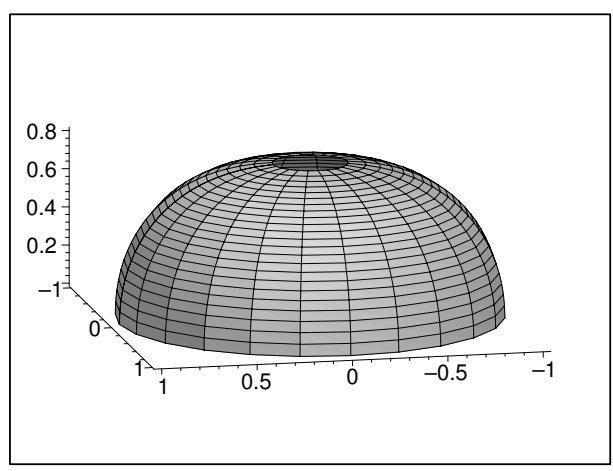

Figure 9: A dome very close to the Mylar dome, $\varepsilon=1000000$.

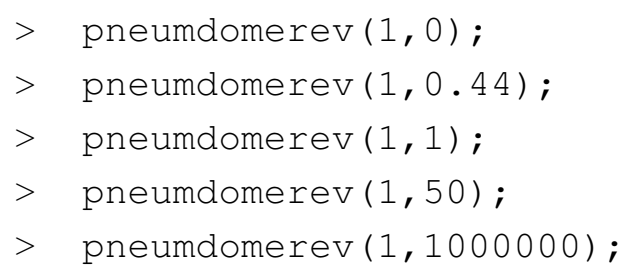

The reader will note that the tops of the domes do not appear. This is not simply a product of numerically solving the differential equation (23). Kawaguchi notes that the meridian tension must approach a singularity at the top of the dome, so there is real trouble in trying to solve the differential equation. Of course, for real domes, the extensibility of the fabric allows some curvature. 


\section{Delaunay Surfaces}

In what follows we will continue to neglect the film weight contribution (i.e., we will suppose again that $w(s) \equiv 0$ ), and in that case we obtain from equations (4) and (5),

$$
\begin{aligned}
\frac{\mathrm{d}\left(\sigma_{m}(s) r(s)\right)}{\mathrm{d} s} & =-\sigma_{c}(s) \sin \theta(s) \\
\left(\sigma_{m}(s) r(s)\right) \frac{\mathrm{d} \theta(s)}{\mathrm{d} s} & =-\sigma_{c}(s) \cos \theta(s)+p(s) r(s) .
\end{aligned}
$$

As we said previously, a solution is obtained if $\sigma_{m}(s)=\sigma_{c}(s)=\sigma$ are constant, and then the mean curvature is given by

$$
H(s)=\frac{p(s)}{2 \sigma} .
$$

Furthermore, if we can arrange that the hydrostatic pressure is also a constant (i.e., $p(s)=p_{o}=$ constant), then we end up with a surface of revolution of constant mean curvature

$$
H=\frac{p_{o}}{2 \sigma}=\lambda=\text { constant. }
$$

Such a surface is called a surface of Delaunay.

Using the definition of $\lambda$ and writing (25) as $r r^{\prime} \theta^{\prime}=-r^{\prime} \cos \theta+2 \lambda r r^{\prime}$, integration of the system gives us a geometrical relation (in which $\mu$ is some integration constant)

$$
\cos \theta(s)=\lambda r(s)+\frac{\mu}{r(s)}
$$

that can be recognized as the Gauss map of the Delaunay surfaces (for details see [8, Section 2]). Without any loss of generality we can assume that $\lambda>0$, relying either on physical experiments with membranes and balloons or taking into account the mathematical fact that $r \equiv r(s)$ is always positive and that we can measure $\theta \equiv \theta(s)$ only in two ways - clockwise or counterclockwise. The case when $\lambda \equiv 0$ will be treated separately below.

Differentiating (28) twice with respect to $s$ and using $r^{\prime}=-\sin \theta$, we get

$$
\theta^{\prime}=\lambda-\frac{\mu}{r^{2}}
$$

and

$$
\theta^{\prime \prime}=-\frac{2 \mu}{r^{3}} \sin \theta
$$


Taking into account that $\theta^{\prime}(s)$ is the curvature $\kappa \equiv \kappa(s)=k_{\mu}(s)$ of the profile curve of the surface in the $x z$-plane, (30) can be rewritten in the form

$$
\kappa^{\prime}=2(\lambda-\kappa) \sqrt{\frac{\lambda-\kappa}{\mu}-(2 \lambda-\kappa)^{2}}
$$

where we have chosen the negative square root. This is the intrinsic equation of the meridian curve we seek. By introducing a new variable, $\xi=\lambda-\kappa$, the corresponding integral reduces to [7, Integral 380.111] and we obtain the following solution

$$
\kappa(s)=\lambda \frac{1-4 \lambda \mu+\sqrt{1-4 \lambda \mu} \sin (2 \lambda s)}{1-2 \lambda \mu+\sqrt{1-4 \lambda \mu} \sin (2 \lambda s)}, \quad-\infty \leq \mu \leq \frac{1}{4 \lambda}
$$

which further implies (via (29) and various algebraic manipulations) that

$$
r(s)=\frac{\sqrt{1-2 \lambda \mu+\sqrt{1-4 \lambda \mu} \sin (2 \lambda s)}}{\lambda \sqrt{2}} .
$$

Having this in hand, the Gauss map (i.e., equation (28)) tell us that

$$
\cos \theta(s)=\frac{1+\sqrt{1-4 \lambda \mu} \sin (2 \lambda s)}{\sqrt{2} \sqrt{1-2 \lambda \mu+\sqrt{1-4 \lambda \mu} \sin (2 \lambda s)}}
$$

while (30) leads to

$$
\sin \theta(s)=-\frac{\sqrt{1-4 \lambda \mu} \cos (2 \lambda s)}{\sqrt{2} \sqrt{1-2 \lambda \mu+\sqrt{1-4 \lambda \mu} \sin (2 \lambda s)}} \cdot
$$

Inserting the above expressions into (1) and integrating produces (33), (as, of course, should be the case!) and we have the following.

Theorem 10. Delaunay surfaces are given by $(r(s) \cos v, r(s) \sin v, z(s))$ with

$$
r(s)=\frac{\sqrt{1-2 \lambda \mu+\sqrt{1-4 \lambda \mu} \sin (2 \lambda s)}}{\lambda \sqrt{2}}
$$

and

$$
z(s)=\frac{\mu}{m(\lambda, \mu)} F\left(\lambda s-\frac{\pi}{4}, k\right)+\frac{m(\lambda, \mu)}{\lambda} E\left(\lambda s-\frac{\pi}{4}, k\right)
$$

where $F$ and $E$ are elliptic integrals of the first and second kind respectively and

$$
m(\lambda, \mu)=\frac{\sqrt{1-2 \lambda \mu+\sqrt{1-4 \lambda \mu}}}{\sqrt{2}}, \quad k=\sqrt{\frac{2 \sqrt{1-4 \lambda \mu}}{1-2 \lambda \mu+\sqrt{1-4 \lambda \mu}}} .
$$


Now consider the case when the differential hydrostatic pressure across the membrane vanishes (i.e., $\lambda \equiv 0$ ). In this case, the intrinsic equation (31) reduces to

$$
\tilde{\kappa}^{\prime}=-2 \tilde{\kappa} \sqrt{-\frac{\tilde{\kappa}}{\mu}-\tilde{\kappa}^{2}}
$$

and its solution is

$$
\tilde{\kappa}(s)=-\frac{\mu}{s^{2}+\mu^{2}} .
$$

This time (29) and (28) produce

$$
\tilde{r}(s)=\sqrt{s^{2}+\mu^{2}} \quad \text { and } \quad \tilde{\mathrm{z}}(\mathrm{s})=\mu \ln \left(\frac{\mathrm{s}+\sqrt{\mathrm{s}^{2}+\mu^{2}}}{\mu}\right) .
$$

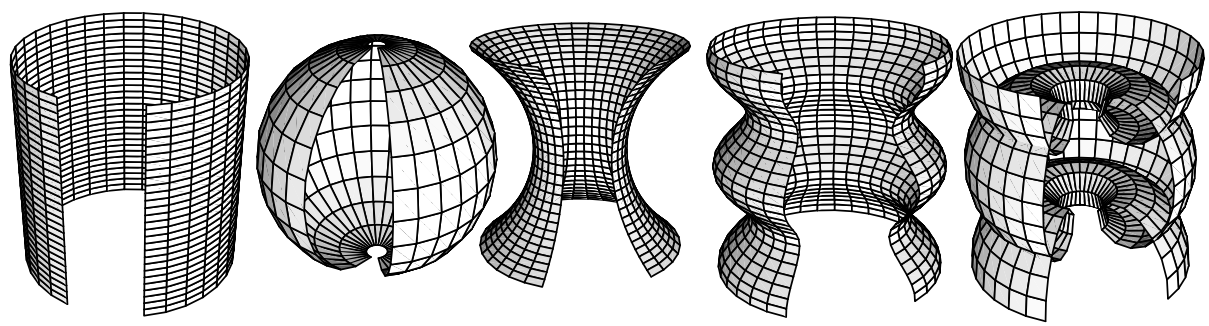

Figure 10: The open parts of the cylinder, sphere, catenoid, unduloid and nodoid shown here are drawn via the profile curves (33) and (37) or (41) and various combinations of the parameters $\lambda$ and $\mu$.

\section{Some Useful Formulas}

The formulas given in the previous section are easily checked in certain cases: by taking $\mu=\frac{1}{4 \lambda}$ we get a cylinder, and by taking $\mu=0$ we get a sphere. The intermediate cases when $0<\mu<\frac{1}{4 \lambda}$ generate unduloids and the ones with $\mu<0$ lead to nodoids. The cases $\lambda=0, \mu>0$ give catenoids. The whole family of non-trivial Delaunay surfaces (omitting the plane) is depicted in Fig. 10.

However, having the explicit form of the parametrization of these surfaces provided by Theorem 10 (and (41)), one can find, in principle, any other geometric 
characteristic of the surface. This follows by a general theorem in classical differential geometry which says that such geometric characteristics are determined by the first and the second fundamental forms of the surface (having respective coefficients $(E, F, G)$ and $(L, M, N))$.

Actually, in our case it is quite easy to see that $E=1, F=0, G=r^{2}(s)$, $L=\theta^{\prime}(s), M=0$ and further direct computations (in the case $\lambda \neq 0$ ) produce

$$
N=\frac{1+\sqrt{1-4 \lambda \mu} \sin (2 \lambda s)}{2 \lambda}
$$

and

$$
k_{\pi}(s)=\lambda \frac{1+\sqrt{1-4 \lambda \mu} \sin (2 \lambda s)}{1-2 \lambda \mu+\sqrt{1-4 \lambda \mu} \sin (2 \lambda s)} .
$$

In the case of the catenoids, one has $E=1, F=0, G=s^{2}+\mu^{2}$ and $L=$ $-\frac{\mu}{s^{2}+\mu^{2}}, M=0, N=\mu$.

If we are interested in the solution of the inverse problem (i.e., how to find the corresponding parameters $\lambda, \mu$ if the maximal $r_{\max }$ and minimal $r_{\min }$ distances from the symmetry axis are given), then we can see that, in the case of unduloids, we have

$$
\begin{aligned}
\lambda=\frac{1}{r_{\text {max }}+r_{\text {min }}}, & \mu=\frac{r_{\text {max }} r_{\text {min }}}{r_{\text {max }}+r_{\text {min }}} \\
r_{\max }=\frac{\sqrt{1-2 \lambda \mu+\sqrt{1-4 \lambda \mu}}}{\lambda \sqrt{2}}, & r_{\min }=\frac{\sqrt{1-2 \lambda \mu-\sqrt{1-4 \lambda \mu}}}{\lambda \sqrt{2}}
\end{aligned}
$$

and the respective nodoid with the same geometric data can be built with

$$
\lambda=\frac{1}{r_{\max }-r_{\min }}, \quad \mu=-\frac{r_{\max } r_{\min }}{r_{\max }-r_{\min }} .
$$

The parameters for the cylinders and spheres are recovered directly via (44), taking into account their geometries specified respectively by $r_{\max }=r_{\min }$ for cylinders and $r_{\max } \in \mathbb{R}^{+}, r_{\min }=0$ for spheres. Analogously, by taking $\mu=r_{\min }>0$ in (41), we recover the explicit parameterization of the catenoid whose neck radius is $r_{\text {min }}$. Notice also that we can find $\theta(s)$ in explicit form. In the case $\lambda \neq 0$, we have

$$
\begin{aligned}
\theta(s) & =\lambda s+\arctan \frac{\sqrt{1-4 \lambda \mu}}{2 \lambda \mu}-\arctan \frac{\sqrt{1-4 \lambda \mu}+(1-2 \lambda \mu) \tan (s \lambda)}{2 \lambda \mu} \\
& =\arcsin \frac{\sqrt{1-4 \lambda \mu}}{\sqrt{2} \sqrt{1-2 \lambda \mu}}-\arcsin \frac{\sqrt{1-4 \lambda \mu} \cos (2 s \lambda)}{\sqrt{2} \sqrt{1-2 \lambda \mu+\sqrt{1-4 \lambda \mu} \sin (2 s \lambda)}}
\end{aligned}
$$




$$
\begin{aligned}
& =\arccos \frac{1}{\sqrt{2} \sqrt{1-2 \lambda \mu}}-\arccos \frac{1+\sqrt{1-4 \lambda \mu} \sin (2 s \lambda)}{\sqrt{2} \sqrt{1-2 \lambda \mu+\sqrt{1-4 \lambda \mu} \sin (2 s \lambda)}} \\
& =\arctan \sqrt{1-4 \lambda \mu}-\arctan \frac{\sqrt{1-4 \lambda \mu} \cos (2 s \lambda)}{1+\sqrt{1-4 \lambda \mu} \sin (2 s \lambda)}
\end{aligned}
$$

of which the first is obtained by a direct integration of (29) and the rest are results of purely trigonometric considerations. The above expressions comply with the initial condition

$$
\theta(0)=0
$$

The real value of these formulas however is that they provide (probably new) quite interesting relationships between the well known transcendental functions of the classical analysis.

For the catenoids we have

$$
\tilde{\theta}(s)=-\arctan \frac{s}{\mu}
$$

and obviously the initial condition

$$
\tilde{\theta}(0)=0
$$

is satisfied as well. By inverting (47) we find $s=-\mu \tan \tilde{\theta}$, and substituting in (41), we obtain the profile curve of the so-called Whewell parameterization (see $[25,26]$ ). The profile curves of the unduloid and the nodoid surfaces can be written (at least in the vicinity of the origin) in this parameterization as well. The abovementioned parameterization of the Mylar balloon can be found in [12].

All the formulas above are also indispensable in problems such as finding the length, lateral surface area or the volume inside arbitrarily chosen segments of Delaunay surfaces (for more details see [10] and [11]). The following is a Maple procedure for creating Delaunay surfaces from the parametrization above. We list some examples (while omitting pictures) that the reader can try.

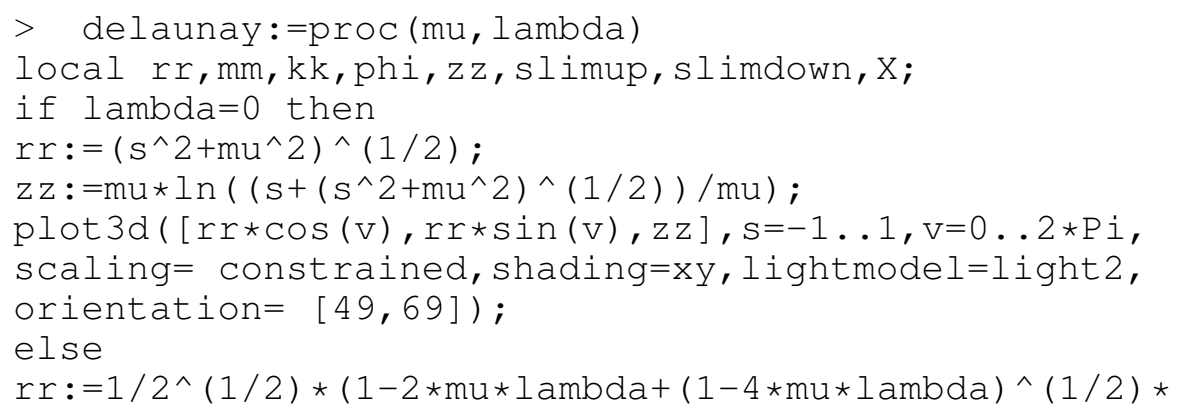




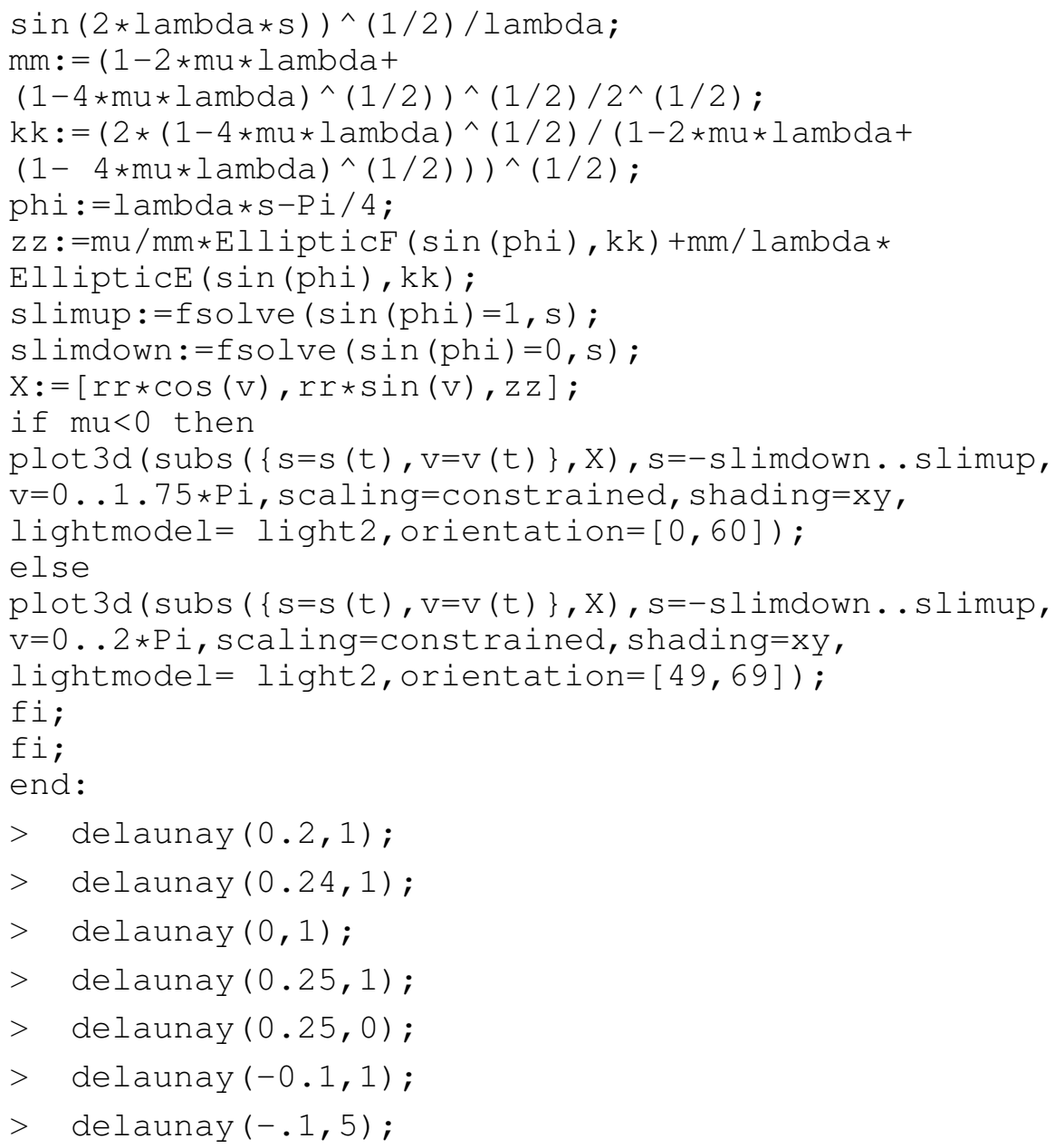

\section{The case $w \neq 0$}

Up until now, we have considered the equilibrium equation

$$
\frac{\partial}{\partial s}\left(\sigma_{m}(s) r(s) \mathbf{t}(s, v)\right)-\sigma_{c}(s) \mathbf{e}_{1}(v)+p(s) r(s) \overline{\mathbf{n}}(s, v)-w(s) r(s) \mathbf{k}=0
$$

under the assumption that $w=0$. In this final section, we want to give the reader an idea of the practical implications inherent in considering $w \neq 0$. More specifically, we will consider a model for large scientific balloons. The model we describe may be found in [4] (with the convention $r^{\prime}=\sin \theta$ instead of ours) where 
a shooting method for finding numerical solutions is given. We shall present an alternative interactive Maple approach to finding solutions of this model.

Definition 11. If the hoop stress $\sigma_{c}$ is identically zero for a "balloon-shape", then the resulting surface is called a natural shape.

We have seen previously that the Mylar balloon and Kawaguchi's domes are natural shapes. Here, let's look at natural shape large scientific balloons. These balloons can be as large as 40 million cubic feet in volume with surface area of around 20 acres (see [4]). Such a balloon can carry a load of 5000 pounds to an altitude of 126000 feet.

Now, as in Subsection 2.2, we can project (49) in particular directions to obtain defining equations for a model. Here, taking $\sigma_{c}=0$, we project onto the $\mathbf{k}$ and $\mathbf{e}_{1}$ directions (omitting details) to obtain the respective equations:

$$
\begin{aligned}
\frac{\partial}{\partial s}\left(\sigma_{m} r \cos \theta\right)+p r \sin \theta-w r & =0 \\
-\frac{\partial}{\partial s}\left(\sigma_{m} r \sin \theta\right)+p r \cos \theta & =0 .
\end{aligned}
$$

After carrying out the differentiation in the first terms, we obtain the following.

$$
\begin{gathered}
\frac{\mathrm{d}\left(\sigma_{m} r\right)}{\mathrm{d} s} \cos \theta-\sigma_{m} r \sin \theta \frac{\mathrm{d} \theta}{\mathrm{d} s}+p r \sin \theta-w r=0 \\
-\frac{\mathrm{d}\left(\sigma_{m} r\right)}{\mathrm{d} s} \sin \theta-\sigma_{m} r \cos \theta \frac{\mathrm{d} \theta}{\mathrm{d} s}+p r \cos \theta=0 .
\end{gathered}
$$

Then, considering this system as a pair of simultaneous equations in the variables, $\mathrm{d}\left(\sigma_{m} r\right) / \mathrm{d} s$ and $\mathrm{d} \theta / \mathrm{d} s$, we can solve to obtain the following

$$
\frac{\mathrm{d} \theta}{\mathrm{d} s}=-\frac{w \sin \theta}{\sigma_{m}}+\frac{p}{\sigma_{m}}, \quad \frac{\mathrm{d}\left(\sigma_{m} r\right)}{\mathrm{d} s}=w r \cos \theta .
$$

There are two added ingredients for the model. First, we recognize that the shape of a balloon changes as it ascends until it reaches a float altitude where the buoyancy force balances gravity. This produces the formula $w=w_{d} \rho(s)$ for the weight density of the balloon, where the subscript " $d$ " will always refer to the situation at the float altitude. Furthermore, the function $\rho(s)$ can be taken to be $\rho(s)=r_{d}(s) / r(s)$, where $r_{d}(s)$ is the radius function for the float shape. We also can identify the pressure function in the balloon case as $p(z)=b(z+a)$, where $b$ is the buoyancy and $a$ is the distance from the zero-pressure level to the bottom 
of the balloon. (This is why these balloons are sometimes called zero-pressure natural shape balloons.)

In order to get numerical solutions to this system of equations, it is convenient to non-dimensionalize as follows (see [4]). Archimedes's principle says that $w_{d} A_{d}+$ $L=b_{d} V_{d}$; that is, at float, the total weight of the balloon is balanced by the buoyancy times the volume displaced. Of course, the gross weight $G=w_{d} A_{d}+L$ is the same at all altitudes. Divide by the load $L$ to get the unitless quantity $\left(w_{d} / L\right) A_{d}+1=\left(b_{d} / L\right) V_{d}$. Hence, $b_{d} / L$ has units of $1 /$ length $^{3}$ and if we let

$$
\lambda=\left(\frac{L}{b_{d}}\right)^{1 / 3}, \quad \Sigma=(2 \pi)^{1 / 3} \frac{w_{d}}{b_{d} \lambda}
$$

then $\lambda$ has units of length and we can non-dimensionalize by defining:

$$
\bar{a}=\frac{a}{\lambda}, \quad \bar{r}=\frac{r}{\lambda}, \quad \bar{z}=\frac{z}{\lambda}, \quad \bar{s}=\frac{s}{\lambda}, \quad \widehat{\Sigma}=\frac{\Sigma}{(2 \pi)^{1 / 3}}=\frac{w_{d}}{b_{d} \lambda}, \quad \bar{\sigma}_{m}=\frac{\sigma_{m}}{b_{d} \lambda^{2}} .
$$

We further note that

$$
\frac{\mathrm{d} \bar{s}}{\mathrm{~d} s}=\frac{1}{\lambda}, \quad \frac{\mathrm{d} \theta}{\mathrm{d} s}=\frac{\mathrm{d} \theta}{\mathrm{d} \bar{s}} \frac{1}{\lambda}, \quad \frac{\mathrm{d} r}{\mathrm{~d} s}=\frac{\mathrm{d} r}{\mathrm{~d} \bar{s}}, \quad \frac{\mathrm{d} z}{\mathrm{~d} s}=\frac{\mathrm{d} z}{\mathrm{~d} \bar{s}}
$$

and that $\rho$ is invariant under this scaling. Putting these into (54) gives

$$
\frac{\mathrm{d} \theta}{\mathrm{d} \bar{s}}=-\frac{\widehat{\Sigma} \rho \bar{r} \sin \theta}{\bar{\sigma}_{m} \bar{r}}+\frac{\tau_{b} \bar{r}(\bar{z}+\bar{a})}{\bar{\sigma}_{m} \bar{r}}, \quad \frac{\mathrm{d}\left(\bar{\sigma}_{m} \bar{r}\right)}{\mathrm{d} \bar{s}}=\widehat{\Sigma} \rho \bar{r} \cos \theta
$$

where $\tau_{b}=b / b_{d}$ is the relative buoyancy. Note that, at float, $\tau_{b}=1, \rho=1$ and, since it will be very small, $a=0$.

Now define $\bar{m}=1 /\left(\sigma_{m} \bar{r}\right)$ and calculate

$$
\frac{\mathrm{d} \bar{m}}{\mathrm{~d} \bar{s}}=-\widehat{\Sigma} \rho \bar{r} \bar{m}^{2} \cos \theta
$$

to obtain the following final set of model equations for the natural shape balloon (where we now drop the bars above the variables).

Theorem 12 ([4]) The following system models the natural shape balloon.

$$
\begin{aligned}
\frac{\mathrm{d} \theta}{\mathrm{d} s} & =-\widehat{\Sigma} \rho r m \sin \theta+m \tau_{b} r(z+a) \\
\frac{\mathrm{d} m}{\mathrm{~d} s} & =-\widehat{\Sigma} \rho r m^{2} \cos \theta
\end{aligned}
$$




$$
\begin{aligned}
& \frac{\mathrm{d} r}{\mathrm{~d} s}=-\sin \theta \\
& \frac{\mathrm{d} z}{\mathrm{~d} s}=\cos \theta
\end{aligned}
$$

with initial conditions

$$
\theta(0)=-\theta_{0}, \quad m(0)=2 \pi \cos \theta_{0}, \quad r(0)=0, \quad z(0)=0
$$

and boundary conditions

$$
\theta(\ell)= \pm \pi / 2, \quad r(\ell)=0
$$

where $\ell$ is the length of the meridian curve on the balloon (which we assume to be constant during ascent to float).

Proof: We have shown the derivation of the equations already and explained the negative in the initial angle. The boundary conditions arise from physical considerations; namely, we want the balloon to be closed and unwrinkled at the top, so it must be "flat" there. The second initial condition arises as follows. The total film load at $s=0$ is $L / \cos \theta_{0}$ since the load $L$ is attached to the bottom of the balloon. This stress is distributed around a parallel circle, so we have $L / \cos \theta_{0}=2 \pi\left(\sigma_{m} r\right)(0)$. Now we obtain,

$$
\bar{m}(0)=\frac{1}{\left(\bar{\sigma}_{m} \bar{r}\right)(0)}=\frac{1}{\left(\frac{\sigma_{m}}{b_{d} \lambda^{2}} \cdot \frac{r}{\lambda}\right)(0)}=\frac{b_{d} \lambda^{3}}{\left(\sigma_{m} r\right)(0)}=\frac{L}{\frac{L}{2 \pi \cos \theta_{0}}}=2 \pi \cos \theta_{0} .
$$

As stated in [4], in practice, $\ell$ and the float shape are known at the start, so $\rho(s)=r_{d}(s) / r(s)$ is defined in terms of $r(s)$ and $\tau_{b}$ is determined by the altitude. Therefore, a shooting method can be used to solve the system while satisfying the boundary conditions. The results of just such a shooting method are given in [4]. Here we give a simple Maple procedure which allows the user to change $a$ and $\theta_{0}$ interactively to satisfy the boundary conditions (61). The examples we give are chosen simply for comparison with the results of [4]. The method consists of two parts. We first use Maple to solve the system of Theorem 12 numerically for the float shape: that is, the shape with $\tau_{b}=1, \rho=1$ and $a=0$. This gives the $r_{d}(s)$ that allows us to define $\rho(s)$ for the ascending balloon. Note that we still need to interactively satisfy the boundary conditions here to obtain the float shape. Once we have the correct $\theta_{0}$ for float, we can feed this into the general system, thus defining $\rho(s)$, and interactively determine $\theta_{0}$ and $a$ for the ascending balloon so 
that the boundary conditions are satisfied. Here is the procedure and examples (see also the Figs. 11-16).

$>$ with(plots):with (LinearAlgebra):

The following are the float equations.

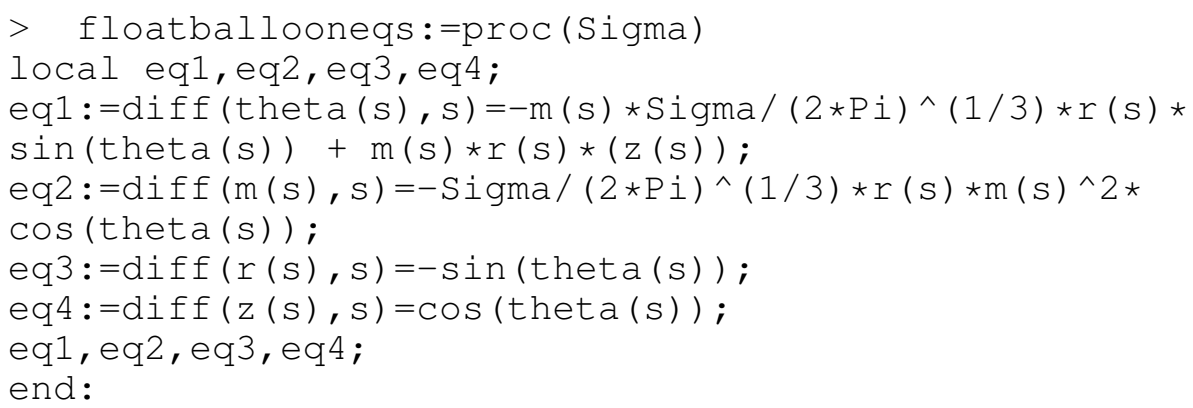

These can now be solved numerically for different $\theta_{0}$ 's. Of course, the correct $\theta_{0}$ satisfies the boundary conditions (61) and so we interactively search for this $\theta_{0}$. The interactive nature of Maple allows for much easier programming.

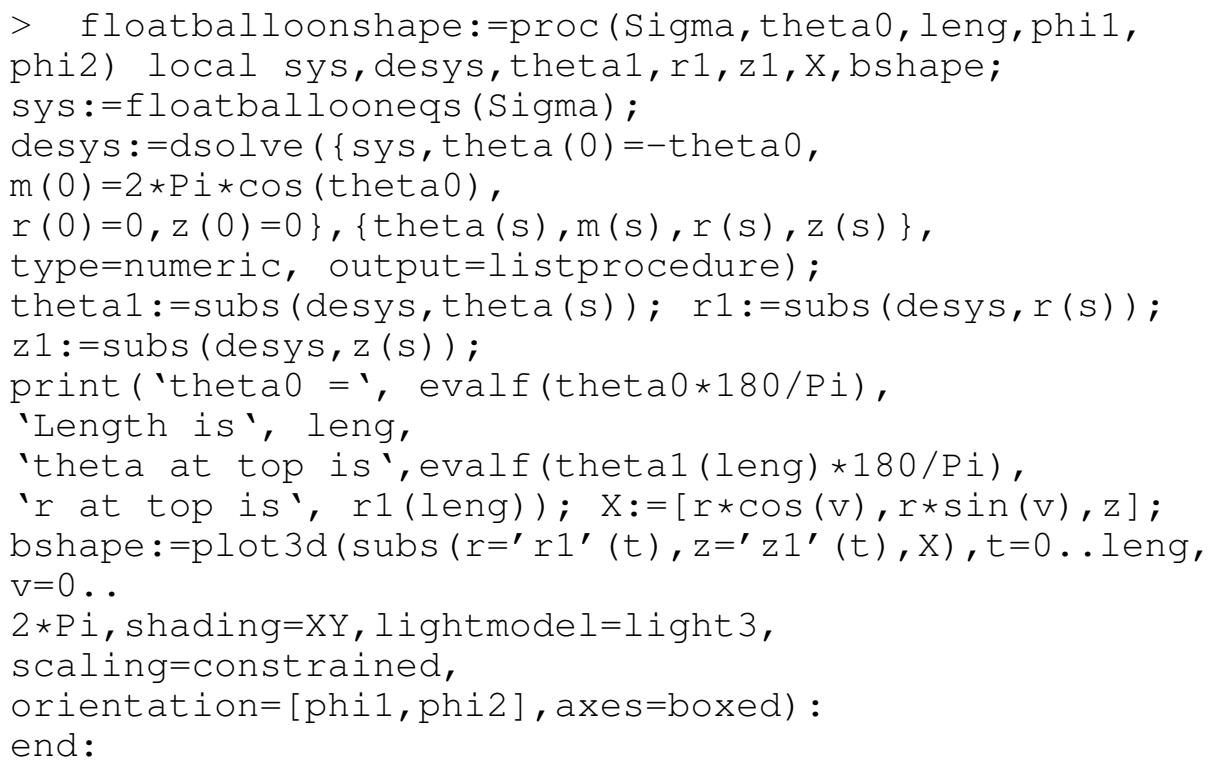

Here are some float shapes for the reader to explore. We provide output only for the case $\Sigma=0.4$ below however.

$>$ floatballoonshape $(0.01,0.882415,2.009001055,0,90)$; 


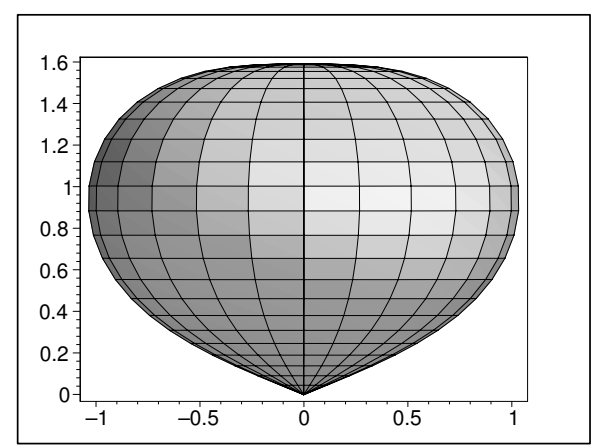

Figure 11: Balloon at float, $\Sigma=0.4$.

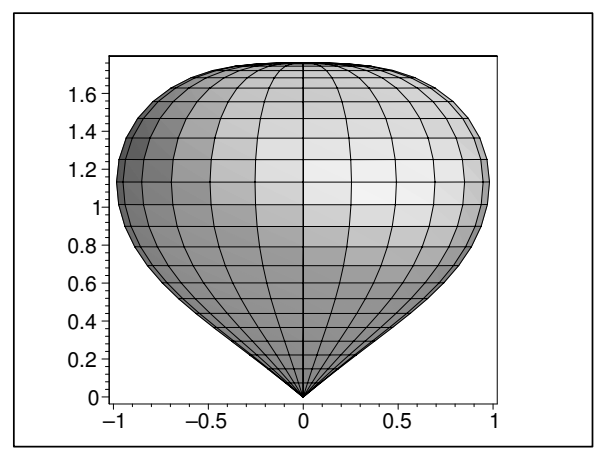

Figure 12: Balloon right before float, $\Sigma=0.4, \tau=1.1$.

\footnotetext{
$>$ floatballoonshape $(0.1,0.94931,2.154547,0,90)$;

$>$ floatballoonshape (0.4, evalf(67.9215*Pi/180),

$2.8706,0,90)$;

$>$ floatballoonshape $(0.8,1.41261,4.4009637,0,90)$;
}

Now we look at some ascent shapes. We begin with the float shape to determine what is called "floattheta0" in the procedure.

$>$ ascentshape $:=$ proc (Sigma, tau, a, floattheta 0 , theta 0 , leng, phil,phi2)

local Feq1, Feq2, Feq3, Feq4, Fsys, Fdesys, Fr1, eq1, eq2, eq3, eq4, sys, desys, theta1, r1, z1, X, bshape;

Feq1:=diff $($ theta $(s), s)=-m(s) \star S i g m a /(2 * P i) \wedge(1 / 3) \star r(s) \star$ $\sin ($ theta $(\mathrm{s}))+\mathrm{m}(\mathrm{s}) * \mathrm{r}(\mathrm{s}) *(\mathrm{z}(\mathrm{s}))$;

Feq2:=diff $(\mathrm{m}(\mathrm{s}), \mathrm{s})=-\mathrm{Sigma} /(2 \star \mathrm{Pi}) \wedge(1 / 3) \star r(\mathrm{~s}) \star \mathrm{m}(\mathrm{s}) \wedge 2 \star$ $\cos ($ theta $(\mathrm{s}))$;

Feq3 $:=\operatorname{diff}(r(s), s)=-\sin ($ theta $(s))$;

Feq4: $=\operatorname{diff}(z(s), s)=\cos ($ theta $(s))$;

Fsys:=Feq1, Feq2, Feq3, Feq4;

Fdesys: $=$ dsolve $(\{$ Esys, theta $(0)=-$ floattheta $0, \mathrm{~m}(0)=$

$2 \star P i \star \cos (f l o a t t h e t a 0), r(0)=0, z(0)=0\},\{$ theta $(s)$,

$\mathrm{m}(\mathrm{s}), r(\mathrm{~s}), \mathrm{z}(\mathrm{s})\}$, type=numeric, output=listprocedure) ;

Frl:=subs (Edesys, r (s)) ;

eq1:=diff $($ theta $(s), s)=-m(s) * \operatorname{Sigma} /(2 * P i) \wedge(1 / 3) * \operatorname{Frl}(s)$

* $\sin (\operatorname{theta}(\mathrm{s}))+\operatorname{tau} * \mathrm{~m}(\mathrm{~s}) * \mathrm{r}(\mathrm{s}) *(\mathrm{z}(\mathrm{s})+\mathrm{a})$;

eq2 : =diff $(\mathrm{m}(\mathrm{s}), \mathrm{s})=-\operatorname{Sigma} /(2 * \mathrm{Pi}) \wedge(1 / 3) \star \operatorname{Fr} 1(\mathrm{~s}) \star \mathrm{m}(\mathrm{s}) \wedge 2 *$

cos (theta (s));

eq3:=diff $(r(s), s)=-\sin ($ theta $(s))$;

eq4 $:=\operatorname{diff}(z(s), s)=\cos ($ theta $(s))$;

sys:=eq1, eq2, eq3, eq4 ; 


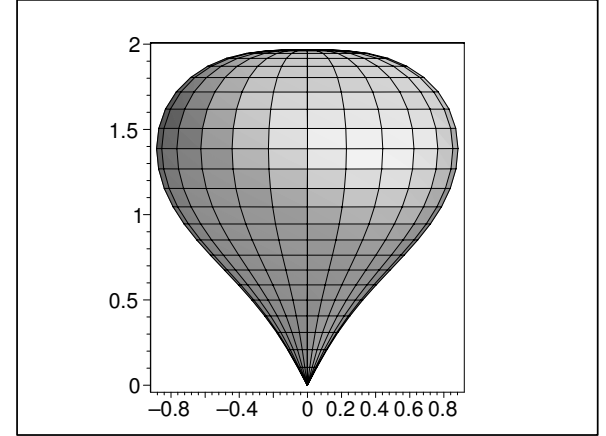

Figure 13: Balloon before float, $\Sigma=0.4, \tau=1.4$.

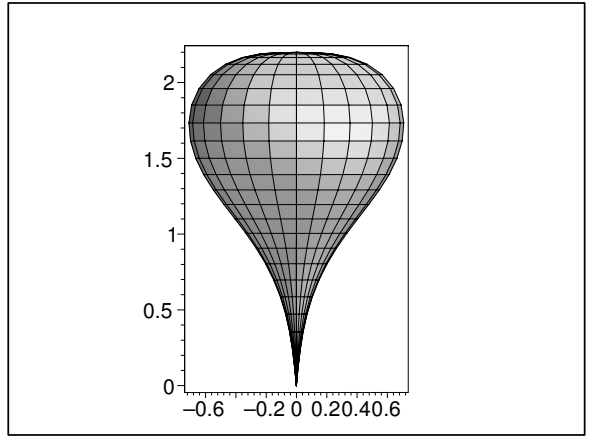

Figure 14: Balloon before float, $\Sigma=0.4, \tau=2.5$.

desys: $=$ dsolve $(\{$ sys, theta $(0)=-$ theta $0, \mathrm{~m}(0)=2 \star \mathrm{P} i *$ $\cos ($ theta 0$), r(0)=0, z(0)=0\},\{\operatorname{theta}(s), m(s), r(s), z(s)\}$, type=numeric, output $=1$ istprocedure) ;

theta1:=subs (desys, theta(s)); r1:=subs (desys,r(s)); $\mathrm{z} 1:=$ subs (desys, z (s)) ;

print ('theta0 =', evalf(theta0*180/Pi), 'Length is', leng, 'theta at top is', evalf(thetal(leng)*180/Pi), 'r at top is', rl(leng));

$X:=[r * \cos (v), r * \sin (v), z]$;

bshape: $=p l$ lot $3 \mathrm{~d}\left(\mathrm{subs}\left(r=^{\prime} r 1^{\prime}(t), z={ }^{\prime} z l^{\prime}(t), X\right), t=0 \ldots\right.$ leng, $\mathrm{V}=0$..

$2 \star P i$, shading $=X Y, l i g h t m o d e l=1$ ight 3 ,

scaling=constrained,

orientation=[phi1, phi2], axes=boxed):

end:

$>$ floatballoonshape(0.4, evalf(67.9215*Pi/180), $2.8706,0,90)$;

theta0 $=, 67.92149998$, Length is, 2.8706, theta at top is, 90.00340531, $r$ at top is, $0.95611135557762718010^{-5}$

$>$ ascentshape (0.4,1.1,-0.47, evalf (67.9215*Pi/180), evalf $(50.9175 * \mathrm{Pi} / 180), 2.8706,0,90)$;

theta0 $=, 50.91750001$, Length is, 2.8706, theta at top is, 90.01011223, $r$ at top is, 0.000234004842540770989 


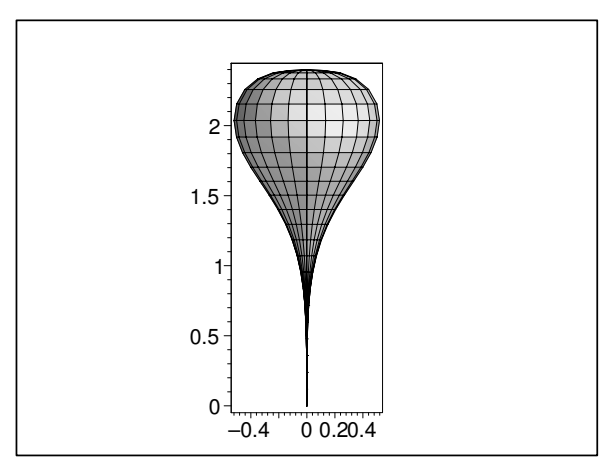

Figure 15: Balloon before float, $\Sigma=0.4, \tau=6$.

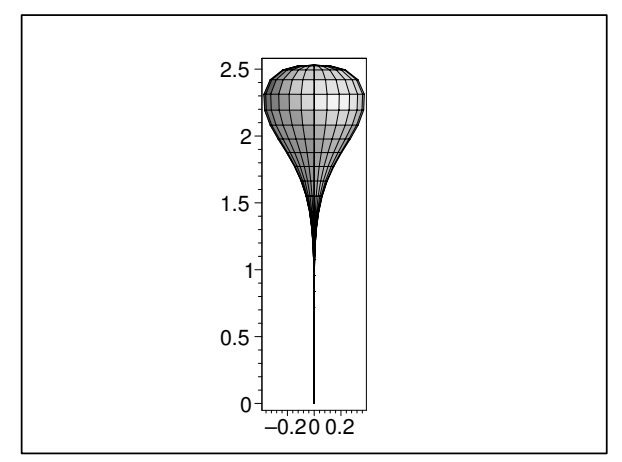

Figure 16: Balloon soon after launch, $\Sigma=0.4, \tau=15$.

$>$ ascentshape $(0.4,1.4,-0.84659$, evalf $(67.9215 * \mathrm{Pi} / 180)$ evalf $(27.60182 * \mathrm{Pi} / 180), 2.8706,0,90)$;

theta0 $=, 27.60181999$, Length is,2.8706, theta at top is,90.00000318, $r$ at top is, 0.0000988085877405243604

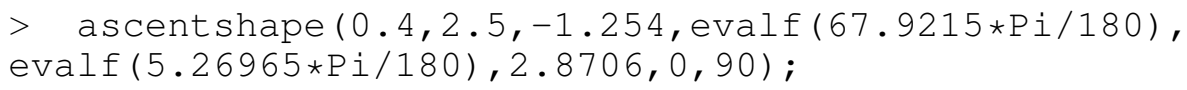

theta0 $=, 5.269650000$, Length is, 2.8706, theta at top is, 90.09962756, $r$ at top is, 0.00115205297864118240

$>$ ascentshape $(0.4,6,-1.6546$, evalf(67.9215*Pi/180), evalf $(0.09758 * \mathrm{Pi} / 180), 2.8706,0,90)$;

theta0 $=, 0.09757999996$, Length is, 2.8706, theta at top is, 90.00008817, $r$ at top is, 0.000993707755309609052

$>\quad$ ascentshape $(0.4,15,-1.96503$, evalf (67.9215*Pi/180), evalf $(0.000069008 * \mathrm{Pi} / 180), 2.8706,0,90)$;

theta0 $=, 0.00006900799997$, Length is, 2.8706,

theta at top is, 90.00603570, $r$ at top is, 0.000134893472887379508 


\section{Appendix: Recollections of Elliptic Functions}

In order to help the reader with certain aspects of this paper, we recall some very basic information about elliptic functions. The easiest way to understand elliptic functions is to consider them as analogues of the ordinary trigonometric functions. Just as the $\arcsin x$ is given by an integral with $\sin t$ the inverse function for that integral, we can construct elliptic functions as inverse functions for certain integrals. Begin by fixing some $k$ with $0 \leq k \leq 1$ (called the modulus).

Definition 13. The Jacobi sine function $\operatorname{sn}(u, k)$ is the inverse function of the following integral:

$$
F(z, k)=\int_{0}^{z} \frac{\mathrm{d} t}{\sqrt{1-t^{2}} \sqrt{1-k^{2} t^{2}}} .
$$

More specifically,

$$
u=\int_{0}^{\operatorname{sn}(u, k)} \frac{\mathrm{d} t}{\sqrt{1-t^{2}} \sqrt{1-k^{2} t^{2}}} .
$$

We call $F(z, k)$ an elliptic integral of the first kind. An elliptic integral of the second kind has the form $E(z, k)$ in

$$
E(z, k)=\int_{0}^{z} \frac{\sqrt{1-k^{2} t^{2}}}{\sqrt{1-t^{2}}} \mathrm{~d} t .
$$

When $z=1$ in $F(z, k)$ and $E(z, k)$, these integrals are respectively denoted by $K(k)$ and $E(k)$ and called the complete elliptic integrals of the first and second kind, respectively.

The Jacobi cosine function $\operatorname{cn}(u, k)$ may be defined in terms of $\operatorname{sn}(u, k)$ by means of an identity (with square root taken appropriately):

$$
\operatorname{sn}^{2}(u, k)+\operatorname{cn}^{2}(u, k)=1 \text {. }
$$

A third Jacobi elliptic function $\operatorname{dn}(u, k)$ is defined by the equation

$$
\operatorname{dn}^{2}(u, k)+k^{2} \operatorname{sn}^{2}(u, k)=1 .
$$

The integral definition of $\operatorname{sn}(u, k)$ makes it clear that $\operatorname{sn}(u, 0)=\sin u$. Of course, $\operatorname{cn}(u, 0)=\cos u$ as well. The case $k=1$ gives something interesting as well. Namely, we have $\operatorname{sn}(u, 1)=\tanh u$ and $\operatorname{cn}(u, 1)=\operatorname{sech} u$. 
The derivatives of the elliptic functions can be found from the definitions. For instance, the derivative of $\operatorname{sn}(u, k)$ may be computed as follows. Suppose that $z=z(u)$. Then

$$
\frac{\mathrm{d} F}{\mathrm{~d} u}=\frac{\mathrm{d} F}{\mathrm{~d} z} \frac{\mathrm{d} z}{\mathrm{~d} u}=\frac{1}{\sqrt{1-z^{2}} \sqrt{1-k^{2} z^{2}}} \frac{\mathrm{d} z}{\mathrm{~d} u}
$$

But we know that for $z=\operatorname{sn}(u, k)$ we have $F(z, k)=u$, so, replacing $z$ by $\operatorname{sn}(u, k)$ and using $\mathrm{d} u / \mathrm{d} u=1$, we obtain:

$$
\begin{aligned}
1 & =\frac{1}{\sqrt{1-\operatorname{sn}(u, k)^{2}} \sqrt{1-k^{2} \operatorname{sn}(u, k)^{2}}} \frac{\mathrm{d} \operatorname{sn}(u, k)}{\mathrm{d} u} \\
\frac{\mathrm{d} \operatorname{sn}(u, k)}{\mathrm{d} u} & =\sqrt{1-\operatorname{sn}(u, k)^{2}} \sqrt{1-k^{2} \operatorname{sn}(u, k)^{2}} \\
\frac{\mathrm{d} \operatorname{sn}(u, k)}{\mathrm{d} u} & =\operatorname{cn}(u, k) \operatorname{dn}(u, k) .
\end{aligned}
$$

We also have

$$
\frac{\mathrm{d} \operatorname{cn}(u, k)}{\mathrm{d} u}=-\operatorname{sn}(u, k) \mathrm{dn}(u, k), \quad \frac{\mathrm{d} \operatorname{dn}(u, k)}{\mathrm{d} u}=-k^{2} \operatorname{sn}(u, k) \operatorname{cn}(u, k) .
$$

The functions $\operatorname{sn}(u, k)$ and $\operatorname{cn}(u, k)$ are periodic and such that $K(k)$ is $1 / 4$ of the period of $\operatorname{sn}(u, k)$. Here are two simple examples where elliptic functions arise.

Example 14 (The pendulum equation) Let the angle of a pendulum swing be denoted by $x$. Then it is straightforward to determine the equation of motion: $\ddot{x}+(g / l) \sin x=0$, where $g$ is the acceleration due to gravity and $l$ is the length of the pendulum. Here we take units that give $g / l=1$. The pendulum equation then becomes $\ddot{x}+\sin x=0$. We can multiply by $\dot{x}$ to obtain

$$
\begin{aligned}
\dot{x}(\ddot{x}+\sin (x)) & =0 \\
\dot{x} \ddot{x}+4 \sin \left(\frac{x}{2}\right) \cos \left(\frac{x}{2}\right) \frac{\dot{x}}{2} & =0
\end{aligned}
$$

which leads to

$$
\frac{1}{2} \dot{x}^{2}+2 \sin ^{2}\left(\frac{x}{2}\right)=c
$$

by integrating. (Note that, with a unit mass and using $2 \sin ^{2}(x / 2)=1-\cos (x)$, the last equation expresses the conservation of energy.) Now let $y=\sin (x / 2)$ with $2 \dot{y}=\cos (x / 2) \dot{x}=\sqrt{1-y^{2}} \dot{x}$. Then

$$
4 \dot{y}^{2}=\left(1-y^{2}\right) \dot{x}^{2}=\dot{x}^{2}-\sin ^{2}\left(\frac{x}{2}\right) \dot{x}^{2}=\dot{x}^{2} \cos ^{2}\left(\frac{x}{2}\right)
$$


so

$$
\dot{y}^{2}=\frac{1}{4} \dot{x}^{2} \cos ^{2}\left(\frac{x}{2}\right)
$$

By the first part of the calculation, we have

$$
\frac{1}{4} \dot{x}^{2}=\frac{1}{2} c-\sin ^{2}\left(\frac{x}{2}\right)=\frac{1}{2} c-y^{2}, \quad \cos ^{2}\left(\frac{x}{2}\right)=1-y^{2} .
$$

Hence, $\dot{y}^{2}=\left(A-y^{2}\right)\left(1-y^{2}\right)$, where $A=c / 2$. Taking a square root and separating variables gives

$$
\begin{aligned}
t & =\int_{0}^{y} \frac{\mathrm{d} y}{\sqrt{\left(A-y^{2}\right)\left(1-y^{2}\right)}} \\
& =\int_{0}^{\sqrt{A} u} \frac{\sqrt{A} \mathrm{~d} u}{\sqrt{\left(1-u^{2}\right)\left(1-A u^{2}\right)}} \\
& =\sqrt{A} \cdot F(\sqrt{A} u, \sqrt{A})
\end{aligned}
$$

Example 15 (The ellipse) We parametrize an ellipse by $\alpha(t)=(a \sin t, b \cos t)$, where $0 \leq t \leq 2 \pi$ and $a \geq b$. The arclength integral is then

$$
\begin{aligned}
L & =\int_{0}^{2 \pi} \sqrt{\dot{x}^{2}+\dot{y}^{2}} \mathrm{~d} t \\
& =4 a \int_{0}^{2 \pi} \sqrt{1-\varepsilon^{2} \sin ^{2}(t)} \mathrm{d} t
\end{aligned}
$$

in which $\varepsilon=\sqrt{a^{2}-b^{2}} / a$ is the eccentricity of the ellipse. If we substitute $u=$ $\sin t$ with $\mathrm{d} u / \sqrt{1-u^{2}}=\mathrm{d} t$, we then obtain

$$
L=4 a \int_{0}^{1} \frac{\sqrt{1-\varepsilon^{2} u^{2}}}{\sqrt{1-u^{2}}} \mathrm{~d} u=4 a E(\varepsilon) .
$$

\section{Acknowledgements}

The authors would like to acknowledge the financial support provided by the Bulgarian National Science Foundation under a grant B - 1531/2005. 


\section{References}

[1] Antman S., Nonlinear Problems of Elasticity, 2nd Ed., Springer, New York, 1995.

[2] Baginski F., On the Design and Analysis of Inflated Membranes: Natural and Pumpkin Shaped Balloons, SIAM J. Appl. Math. 65 (2005) 838-857.

[3] Baginski F., Chen Q. and Waldman I., Designing the Shape of a Large Scientific Balloon, Applied Math. Modelling 25 (2001) 953-966.

[4] Baginski F., Collier W. and Williams T., A Parallel Shooting Method for Determining the Natural Shape of a Large Scientific Balloon, Siam J. Appl. Math. 58 (1998) 961-974.

[5] Baginski F. and Winker J., The Natural Shape Balloon and Related Models, Adv. Space Res. 33 (2004) 1617-1622.

[6] Birkhoff G. and de Boor C., Piecewise Polynomial Interpolation and Approximations, In: Proc. General Motors Symposium of 1964, H. Garbedian (Ed), Elsewier, Amsterdam, 1965, pp. 164-190.

[7] Dwight H., Tables of Integrals and Other Mathematical Data, 4th Ed., Macmillan, New York, 1961.

[8] Eells J., The Surfaces of Delaunay, Math. Intelligencer 9 (1987) 53-57.

[9] Flügge W., Stresses in Shells, Springer Verlag, New York, USA (1962).

[10] Grason G. and Santangelo C., Undulated Cylinders of Charged Diblock Copolymers, Eur. Phys. J. E 20 (2006) 335-346.

[11] Hadzhilazova M., Mladenov I. and Oprea J., Unduloids and Their Geometry, Archivum Mathematicum 43 (2007) 417- 429.

[12] Hadzhilazova M. and Mladenov I., Once More the Mylar Balloon, CRAS (Sofia) 61 (2008) 847-856.

[13] Irvine H., Cable Structures, MIT series in Structural Mechanics, MIT Press, MA, 1981.

[14] Jahnke E., Emde F. and Lösch F., Tafeln Höherer Funktionen, Teubner, Stuttgart, 1960.

[15] Kawaguchi M., The Shallowest Possible Pneumatic Forms, Bull. Int. Assoc. Shell Struct. 18 (1977) 3-11.

[16] Koiso M. and Palmer B., Equilibria for Anisotropic Energies and the Gielis Formula, Forma 23 (2008) 1-8. 
[17] Lennon A. and Pellegrino S., Structural Mechanics of Lobed Inflatable Structures, European Conference on Spacecraft Structures, Materials \& Mechanical Testing 2005, Noordwijk, The Netherlands, 10-12 May 2005 (ESA SP-581, August 2005), 12 pp.

[18] Lopez R., On Linear Weingarten Surfaces, Int. J. Math 19 (2008) 439-448.

[19] Mladenov I. and Oprea J., The Mylar Balloon Revisited, Amer. Math. Monthly 110 (2003) 761-784.

[20] Mladenov I. and Oprea J., The Mylar Balloon: New Viewpoints And Generalizations, In: Geometry, Integrability and Quantization, Softex, Sofia, 2007, pp. 246-263.

[21] Oprea J., The Mathematics of Soap Films: Explorations with Maple, American Mathematical Society, Providence, 2000.

[22] Oprea J., Differential Geometry and Its Applications, Classroom Resources, Mathematical Association of America, Washington D. C., 2007.

[23] Pagitz M. and Pellegrino S., Computation of Buckling Pressure of Pumpkin Balloons, 47th AIAA/ASME/ASCE/AHS/ASC Structures, Structural Dynamics, and Materials Conference 1 - 4 May 2006, Newport, Rhode Island, 2006, 22pp.

[24] Pak I., Inflating Polyhedral Surfaces, MIT Preprint, 2006, 37pp.

[25] Whewell W., On the Intrinsic Equation of a Curve, and Its Application, Camb. Phil. Trans. 8 (1849) 659-671.

[26] Whewell W., Second Memoir on the Intrinsic Equation of a Curve and Its Application, Camb. Phil. Trans. 9 (1850)150-156.

Ivaïlo M. Mladenov

Institute of Biophysics

Bulgarian Academy of Sciences

Acad. G. Bonchev Str., Bl. 21

1113 Sofia, BULGARIA

E-mail address: mladenov@bas .bg

John Oprea

Department of Mathematics

Cleveland State University

Cleveland, $\mathrm{OH}$

44115 U.S.A.

E-mail address: j.opreadcsuohio.edu 